\title{
THE SLOAN DIGITAL SKY SURVEY REVERBERATION MAPPING PROJECT: AN INVESTIGATION OF BIASES IN C IV EMISSION LINE PROPERTIES
}

\author{
K. D. Denney ${ }^{1,2,13}$, Keith Horne ${ }^{3}$, Yue Shen ${ }^{4,5}$, W. N. Brandt $6,7,8$, Luis C. Ho ${ }^{9,10}$, B. M. Peterson ${ }^{1,2}$, \\ Gordon T. Richards ${ }^{11}$, J. R. Trump ${ }^{6,14}$, AND J. GE ${ }^{12}$ \\ ${ }^{1}$ Department of Astronomy, The Ohio State University, 140 West 18th Avenue, Columbus, OH 43210, USA; denney@astronomy.ohio-state.edu \\ ${ }^{2}$ Center for Cosmology and AstroParticle Physics, The Ohio State University, 191 West Woodruff Avenue, Columbus, OH 43210, USA \\ ${ }^{3}$ SUPA Physics/Astronomy, University of St. Andrews, St. Andrews KY16 9SS, Scotland, UK \\ ${ }^{4}$ Department of Astronomy, University of Illinois at Urbana-Champaign, Urbana, IL 61801, USA \\ ${ }^{5}$ National Center for Supercomputing Applications, University of Illinois at Urbana-Champaign, Urbana, IL 61801, USA \\ ${ }^{6}$ Department of Astronomy \& Astrophysics, The Pennsylvania State University, 525 Davey Lab, University Park, PA 16802, USA \\ ${ }^{7}$ Institute for Gravitation and the Cosmos, The Pennsylvania State University, University Park, PA 16802, USA \\ ${ }^{8}$ Department of Physics, The Pennsylvania State University, 104 Davey Lab, University Park, PA 16802, USA \\ ${ }^{9}$ Kavli Institute for Astronomy and Astrophysics, Peking University, Beijing 100871, China \\ ${ }^{10}$ Department of Astronomy, School of Physics, Peking University, Beijing 100871, China \\ ${ }^{11}$ Department of Physics, Drexel University, 3141 Chestnut Street, Philadelphia, PA 19104, USA \\ 12 Astronomy Department, University of Florida, 211 Bryant Space Science Center, P.O. Box 112055, Gainesville, FL 32611-2055, USA \\ Received 2016 January 20; accepted 2016 March 16; published 2016 May 26
}

\begin{abstract}
We investigate the dependence on data quality of quasar properties measured from the $\mathrm{C}$ Iv emission line region at high redshifts. Our measurements come from 32 epochs of Sloan Digital Sky Survey Reverberation Mapping Project spectroscopic observations of $482 z>1.46$ quasars. We compare the differences between measurements made from the single-epoch (SE) and coadded spectra, focusing on the $C_{\text {IV }} \lambda 1549$ emission line because of its importance for studies of high-redshift quasar demographics and physical properties, including black hole masses. In addition to statistical errors increasing (by factors of $\sim 2-4$ ), we find increasing systematic offsets with decreasing signal-to-noise ratio $(\mathrm{S} / \mathrm{N})$. The systematic difference (measurement uncertainty) in our lowest-S $/ \mathrm{N}$ $(<5)$ subsample between the $\mathrm{SE}$ and coadded spectrum (i) $\mathrm{C}$ IV equivalent width is $17 \AA$ ( $31 \AA$ ), (ii) centroid wavelength is $<1 \AA$ ( $(\AA)$, and fractional velocity widths, $\Delta V / V$, characterized by (iii) the line dispersion, $\sigma_{l}$, is $0.104(0.12)$, and (iv) the mean absolute deviation (MAD) is $0.072(0.11)$. These remain smaller than the $1 \sigma$ measurement uncertainties for all subsamples considered. The MAD is found to be the most robust line-width characterization. Offsets in the $\mathrm{C}_{\text {IV }} \mathrm{FWHM}$ velocity width and the $\mathrm{C}_{\text {IV }}$ profile characterized by $\mathrm{FWHM} / \sigma_{l}$ are only smaller than the statistical uncertainties when $\mathrm{S} / \mathrm{N}>10$, although offsets in lower-S/N spectra exceed the statistical uncertainties by only a factor of $\sim 1.5$ and may depend on the type of functional fit to the line. Characterizing the $\mathrm{C}$ IV line profile by the kurtosis is the least robust property investigated, as the median systematic coadded-SE measurement differences are larger than the statistical uncertainties for all $\mathrm{S} / \mathrm{N}$ subsamples.
\end{abstract}

Key words: galaxies: active - galaxies: nuclei - quasars: emission lines - quasars: general quasars: supermassive black holes

Supporting material: figure set

\section{INTRODUCTION}

Since their discovery (Schmidt 1963), quasars (synonymously referred to in this work as active galactic nuclei, or AGNs) have evolved from a curiosity to a powerful cosmological probe. They are now understood to be the visible growth phase of the supermassive black holes (BHs) that reside at the center of massive galaxies. The strong correlations between the properties of these BHs and their host galaxies (Kormendy \& Richstone 1995; Magorrian et al. 1998; Ferrarese \& Merritt 2000; Gebhardt et al. 2000a, 2000b; Ferrarese et al. 2001; Tremaine et al. 2002; Wandel 2002; Nelson et al. 2004; Onken et al. 2004; Graham 2007; Bentz et al. 2009b; McConnell \& Ma 2013; see also the review by Kormendy \& Ho 2013 and references therein) strongly suggest that BHs may play an active role in the evolution of galaxies and their surroundings, presumably owing to feedback produced from

\footnotetext{
${ }^{13}$ NSF Astronomy \& Astrophysics Postdoctoral Fellow.

${ }^{14}$ Hubble Fellow.
}

the energy released by the gravitational accretion of material onto the BH (e.g., Hopkins \& Elvis 2010; Debuhr et al. 2011; Fabian 2012). Whether the growth and coevolution of BHs and galaxies are mutually regulating and causal or not (Jahnke \& Macciò 2011; Sun et al. 2015a), these systems remain important probes of the distant and nearby universe.

Many physical properties of the accreting BHs, such as the mass and accretion rates, can be estimated from a single quasar spectrum (e.g., McLure \& Jarvis 2002; Vestergaard 2002; Vestergaard \& Peterson 2006; Wang et al. 2009; Rafiee \& Hall 2011b; Park et al. 2013). This is possible through largely empirical scaling relationships calibrated using small samples of low-redshift AGNs. In particular, reverberation mapping (Blandford \& McKee 1982; Peterson 1993, 2014) provides the calibration for estimating "single-epoch" (SE) quasar masses, using only two observables measurable from a single quasar spectrum: (1) the broad-line region (BLR) velocity, inferred from the velocity width of a broad emission line, and (2) the radius of the variable BLR gas, inferred from the quasar 
luminosity (Laor 1998; Wandel et al. 1999; Kaspi et al. 2000; Bentz et al. 2009a, 2013), and a scale factor, $f$, currently calibrating the AGN BH mass scale through a comparison of the $M_{\mathrm{BH}}-\sigma_{*}$ relationship between AGNs and quiescent BHs (Onken et al. 2004; Woo et al. 2010, 2013; Graham et al. 2011; Park et al. 2012; Grier et al. 2013).

Using quasars for studying cosmology, galaxy evolution, and $\mathrm{BH}$ accretion physics requires an understanding of their evolution in number and mass over cosmic time. This requires measuring these properties for large samples across the universe. In the past several decades, photometric and spectroscopic quasar surveys operating over a wide range of wavelengths and energies have vastly increased the number of known quasars and their redshifts. In particular, the Sloan Digital Sky Survey (SDSS; York et al. 2000) has cataloged $\sim 300,000$ spectroscopically confirmed quasars when combining the fifth edition SDSS Quasar Catalog (Schneider et al. 2010) with the Data Release 10 Quasar catalog (DR10Q; Pâris et al. 2014) from the Baryon Oscillation Spectroscopic Survey (BOSS; Dawson et al. 2013).

Large statistical samples can enable detailed studies of quasar demographics only if the systematic biases associated with survey limits and data quality are well understood. In this study we investigate possible biases in several spectroscopic properties derived for high-redshift quasars due to the low signal-to-noise ratio $(\mathrm{S} / \mathrm{N})$ of typical survey spectra. Since the goal of most surveys is to measure redshifts with which to map the universe, this only requires a high enough $\mathrm{S} / \mathrm{N}$ to reliably detect a high-equivalent-width emission line. Despite the intrinsic high luminosity of quasars, the vast majority will have observed fluxes close to the flux limit of the survey (e.g., Pâris et al. 2014). As a result, the quasar continuum and lower equivalent width (EW) features (such as those used for $\mathrm{BH}$ masses and reliable redshifts) will be at significantly lower $\mathrm{S} / \mathrm{N}$. Consequently, it is important to understand the dataquality limit at which the reliability (defined by both precision and accuracy) of an investigation becomes compromised.

The data used for direct, reverberation-mapping-based $\mathrm{BH}$ measurements are generally of very high quality. This has also become true of the data used to calibrate SE virial $\mathrm{BH}$ mass scaling relations (e.g., Park et al. 2013). As such, our understanding of the general uncertainty in $\mathrm{BH}$ masses derived either directly through reverberation mapping or indirectly through empirical scaling relationships is based on high-quality data. Yet, most of the higher-redshift quasar spectra to which these scaling relations are applied are typically low-S/N, "survey-quality" 15 data. There can be systematic differences between spectroscopic properties measured from the highquality calibration data and the survey-quality data, leading to systematic errors in BH mass estimates. Denney et al. (2009) examined this for $\mathrm{H} \beta$ line-width measurements for two lowredshift, relatively low luminosity AGNs and found statistical and systematic effects that became a significant source of bias for data with spectral $\mathrm{S} / \mathrm{N}<10$ (per pixel) in the continuum.

Here we investigate the effects of low $\mathrm{S} / \mathrm{N}$ on $\mathrm{C}$ IV emissionline properties. These measurable spectroscopic properties are important for direct studies of quasar physics and demographics, as well as the broader evolutionary studies based thereon, as the CIV emission line is present in the optical

\footnotetext{
15 Here we use "survey-quality" to refer to the typically lower spectral S/N ( $\lesssim 3-5$ per pixel at the flux limit) data that are often the product of large, moderate-resolution, flux-limited redshift surveys, such as the SDSS.
}

wavelength regime in the range $1.4 \lesssim z \lesssim 4.8$, which covers the quasar epoch and extends to a time when the universe was less than 1/10 of its current age. The use of $\mathrm{C}$ IV for $\mathrm{SE}$ virial $\mathrm{BH}$ masses remains somewhat controversial throughout the literature owing to apparent inconsistencies between emissionline properties (including FWHM velocity width) and BH mass estimates based on this line and the more robustly tested $\mathrm{H} \beta$ emission line (see many discussions on both sides of the debate by, e.g., Baskin \& Laor 2005; Netzer et al. 2007; Sulentic et al. 2007; Assef et al. 2011; Denney 2012; Shen \& Liu 2012; Runnoe et al. 2013, 2014, and references therein). Nonetheless, $\mathrm{C}$ IV is a particularly important line for these high-redshift studies, as it is the only rest-UV emission line that both has reverberation mapping measurements and has been calibrated for making $\mathrm{SE}$, virial $\mathrm{BH}$ mass estimates.

Our investigation is organized as follows. First, we present the data used for this study in Section 2. Section 3 describes the spectroscopic analysis used to fit and measure the properties of interest. Based on this analysis, we first evaluate statistical and systematic errors in the estimates of the velocity width of the broad $\mathrm{C}_{\text {IV }} \lambda 1549$ emission line. The broad emission line velocity width is one of the two observables needed to estimate a virial $\mathrm{BH}$ mass, which allows subsequent studies of cosmic structure growth, seed BHs, and the coevolution of galaxies and BHs. We also look at other commonly measured $\mathrm{C}_{\mathrm{IV}}$ emission line properties, including the $\mathrm{C}$ IV EW, centroid, and line shape, that may be useful in determining further details of the accretion and feedback processes, structure, and kinematics of the central engine and/or for evaluating other biases in C IVbased BH masses (e.g., Denney 2012). We discuss velocity trends and other results as a function of the data quality for our measured quasar properties in Section 4 and make concluding remarks about our results in Section 5.

\section{C IV SPECTRAL DATA}

In 2014, the SDSS Reverberation Mapping Project (SDSSRM) spectroscopically monitored 849 broad-line quasars in the CFHT-LS W3 field (which is also the AEGIS field and a PanSTARRS medium-deep field) with the BOSS spectrograph (Smee et al. 2013), which is mounted on the SDSS telescope (Gunn et al. 2006) as part of an ancillary program of the SDSS-III surveys (Eisenstein et al. 2011). The SDSS-RM sample covers redshifts over the range $0.1<z<4.5$ down to a flux limit of $i_{\mathrm{psf}}=21.7 \mathrm{mag}$ in a single $7 \mathrm{deg}^{2}$ field. Each of the 32 epochs of observations was a $\sim 2 \mathrm{hr}$ exposure taken during dark/gray time with an average cadence of $\sim 4$ days over a period of 6 months. Further details of the SDSS-RM project, the technical overview, and program goals are provided by Shen et al. (2015).

Here we only consider objects from the SDSS-RM sample with $z>1.46$ so that the C IV emission line and a shortward continuum region are accessible. We further removed three objects for which low-EW emission lines, although clearly seen in the coadded spectrum, were not easily discernible in all or most of the SE spectra, leading the fits (described below) to fail or provide fits that failed to constrain a believable line profile, i.e., fitting only the noise, for most SE spectra. We also removed 12 broad absorption line (BAL) quasars in which the BAL absorbed too much of the $\mathrm{C}$ IV emission line and/or the continuum blueward of $\mathrm{C}$ IV for a reasonable fit to be achieved. Several dozen additional objects $(\sim 10 \%-15 \%$ of our sample) had what we considered "BAL-like" features (although we did 


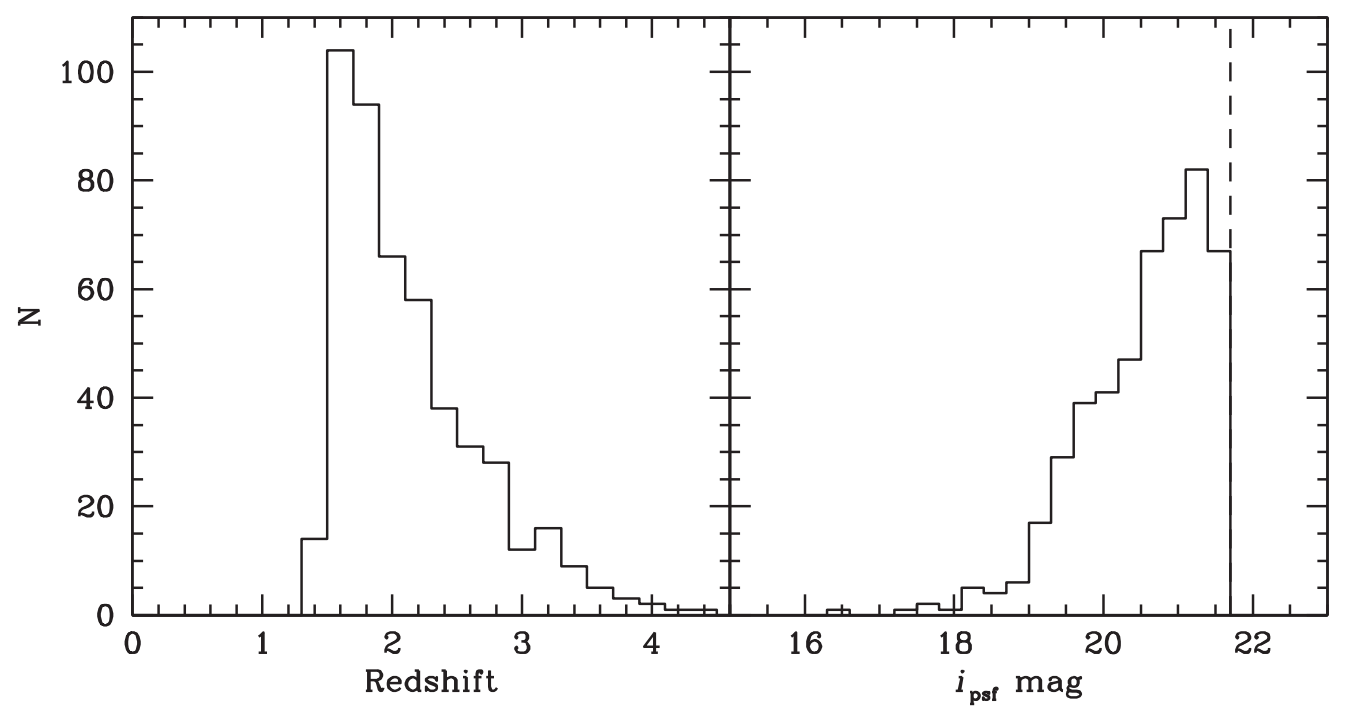

Figure 1. Distribution of redshifts and $i_{\mathrm{psf}}$ magnitudes for the sample of 482 SDSS-RM quasars. The vertical dashed line in the right panel shows the magnitude limit for the SDSS-RM sample.

not set any quantitative measure, e.g., the BALnicity, to define this, and such features also include what appear to be extended regions of unresolved narrow absorption line complexes and "mini-BALs" as opposed to only true BALs), but the continuum between $\mathrm{C}$ IV and $\mathrm{Si}$ IV could still be constrained and a largely symmetric $\mathrm{C}$ iv profile was recovered from the fit, suggesting that the interpolation of the fit over even significant masked absorption regions was as reasonable as could be expected in such cases. We flagged these objects and later compared our analysis with and without their inclusion and find little to no difference in the results-most often, we find the scatter in the distributions marginally smaller when they are excluded. As such, we do not remove any more objects with BAL-like features. Several examples of these types of objects can be found in the supplemental figures.

On occasion, objects were discovered to have "dropped spectra," where the fiber was not properly plugged into the plate. We visually inspected all epochs for all 482 quasars, removing any epoch in which the spectrum was completely absent as a result of this or other problems. We did not initially drop any spectra based on their $\mathrm{S} / \mathrm{N} .{ }^{16}$ However, in a small number of cases, the spectra were too noisy to even detect emission lines, and so these were omitted from subsequent analysis. This occurred most frequently for Epoch 7, the lowest-S/N epoch in the campaign. Our final sample consists of 482 sources. Most (405) QSOs have all 32 epochs of spectra included in our analysis, and only nine QSOs have more than three $(10 \%)$ epochs discarded. Figure 1 shows the redshift and $i_{\text {psf }}$ magnitude distribution of our sample.

\section{SPECTROSCOPIC ANALYSIS}

The SDSS-RM project provides a unique opportunity to study data-quality biases in the measurement of spectroscopic properties of quasars from survey spectra. Each epoch is

\footnotetext{
16 Three out of the 32 epochs have relatively low $\mathrm{S} / \mathrm{N}(\mathrm{S} / \mathrm{N}<0.7\langle\mathrm{~S} / \mathrm{N}\rangle)$ owing to poor observing conditions and/or the inability to obtain the full $2 \mathrm{hr}$ exposure. Sun et al. (2015b) omitted these epochs from their investigation, as they would have systematically biased the measured quasar structure function. We include these low-S/N epochs, as our goal is to analyze spectra over a wide range of $\mathrm{S} / \mathrm{N}$.
}

representative of a typical survey-quality spectrum, although the reader should note that the exposure time of our SE spectra is greater than a typical SDSS or BOSS quasar spectrum, with a $2 \mathrm{hr}$ rather than the minimum 45 -minute exposure. Nonetheless, these longer exposures are offset by our deeper flux limit and so lead to a sample of SE spectra with similar S/N distributions to SDSS or BOSS quasars, where the SDSS spectral $\mathrm{S} / \mathrm{N}$ limits are set by the successive 15-minute exposures being halted when the $\mathrm{S} / \mathrm{N}$ per pixel exceeded 4 for fiber magnitudes of $g=20.2$ and $i=19.9$ and BOSS was similarly limited by exposures stopping when the $(\mathrm{S} / \mathrm{N})^{2}$ exceeded 22 for $i=21$ and 10 for $g=22$. Here we analyze all individual SDSS-RM epochs to measure a distribution of spectral properties expected for SE spectra of a single object. Throughout this work, we use the median of these distributions for each object as our "SE measurement," with the measurement uncertainty defined as half of the 16\%-84\% interpercentile range (HIPR) of the distribution, which would correspond to $1 \sigma$ if the distribution were Gaussian. We then combine all the spectra to make a single, high-S/N, "coadded" spectrum using the latest BOSS spectroscopic pipeline idlspec2d (see Shen et al. 2015, 2016b).

We define the $\mathrm{S} / \mathrm{N}$ per angstrom, determined by the ratio of the mean flux to the dispersion in the flux in an emission-linefree continuum window between rest-frame wavelengths 1690 and $1710 \AA$. The coadded spectra have S/N 5-6 times higher than that of the SE spectra of each object. Figure 2 shows the $\mathrm{S} / \mathrm{N}$ distributions of the SE and coadded spectra for our sample (to compare our sample SE S/N distribution to that of SDSS DR7, see Shen et al. 2011). The median SE (coadded) spectrum continuum $\mathrm{S} / \mathrm{N}$ is 5.5 (25.7), with a scatter, defined by the HIPR, of 4.9 (20.9). Figure 3 shows examples of the C IV emission line region in two SE spectra of SDSS-RM sources, with $\mathrm{S} / \mathrm{N}$ near the sample median (top panels) and the corresponding coadded spectra (bottom panels). The coadded spectrum $\mathrm{S} / \mathrm{N}$ is 48 (46) for the object in the left (right) panel. In both cases, the median $\mathrm{S} / \mathrm{N}$ of all SE spectra of these objects is $\sim 8$, but the SE spectra shown in Figure 3 were chosen from an epoch with an $\mathrm{S} / \mathrm{N}$ close to the median of the full sample $(\mathrm{S} / \mathrm{N} \sim 5)$. 


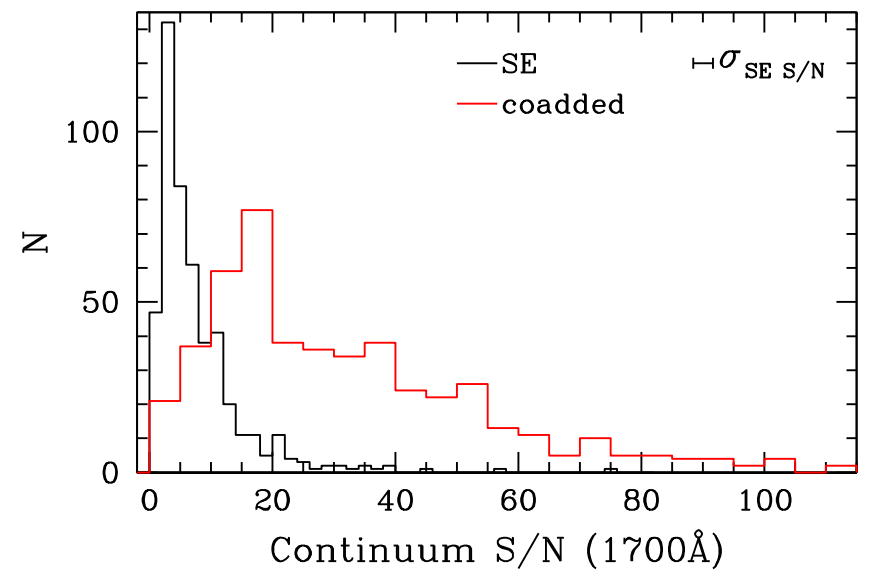

Figure 2. Distribution of S/N of SE spectra (black) and coadded spectra (red) for our sample of 482 SDSS-RM quasars. The coadded $\mathrm{S} / \mathrm{N}$ is measured per angstrom in an emission-line-free continuum window near rest frame $1700 \AA$ of each coadded spectrum, and the SE S/N shown is the median of the distribution of $\mathrm{S} / \mathrm{N}$ measurements made similarly from all good $\mathrm{SE}$ spectra for each object. The $\sigma_{\mathrm{SE}}$ error bar in the upper right corner represents the median of the distribution of scatter measurements made from the SE S/N distributions for all objects. Five coadded spectra have $\mathrm{S} / \mathrm{N}>115$.

Biases in our coadded spectra due to intrinsic variability are unlikely, given the relatively high luminosity of our sample (Vanden Berk et al. 2004; $\mathrm{Zu}$ et al. 2011) and time dilation effects-due to the high redshifts combined with the short campaign period. Our 6-month campaign duration covers only $\sim 1-3$ months in the rest frame of our $z>1.46$ quasars, and quasar (i) variability amplitudes and (ii) reverberation time delays scale with luminosity inversely and directly, respectively. We measured the excess variance, $F_{\text {var }}$, of the flux measured from the $\sim 1700 \AA$ continuum window, assuming a mean spectrophotometric uncertainty of $5 \%$ (see Shen et al. 2015), and find a median value of 0.14 and a scatter of 0.10 . This is consistent with, though somewhat lower than, that measured from the optical continuum for the current RM sample, keeping in mind that the amplitude of UV continuum variability is observed to be greater than that observed in the optical continuum (Kilerci Eser et al. 2015). In any case, such variability on these timescales only acts to change the overall line flux, to first order. Bootstrap Monte Carlo methods are typically applied to RM data to determine the variance in linewidth measurements, primarily taken to be due to variability because the S/Ns of RM sample data are traditionally very high. These find uncertainties in the line widths measured from the mean spectrum on order of a few percent (Collin et al. 2006; Shen et al. 2016a) — too small to affect our results here, assuming the same level of variability effects. Gross profile change or velocity-dependent flux changes have generally been found only to occur on timescales not probed by our campaign (see, e.g., Sergeev et al. 2007; Shen et al. 2015).

\subsection{Line Profile Fits}

Because we are only investigating properties of the C IV emission line in this work, we only fit this localized region of the spectra. Our general procedure for fitting the $\mathrm{C}$ IV emission line follows that described by Denney et al. (2013). We first fit a linear continuum beneath the line, anchored by the mean flux in a blue (typically 1435-1465 $\AA$ ) and a red (typically 1690-1710 $\AA$ ) continuum region on either side of C IV (see
Figure 3). While the AGN continuum is best described by a power law over longer wavelength ranges, there is very little difference locally (i.e., under a single emission-line region) between using a linear continuum and using a power law. We then mask the wavelength regions covering rest frame 1475-1495 $\AA$ and 1600-1680 $\AA$ to exclude possible contributions from any blended N IV] $\lambda 1486$ line emission and the "red shelf" emission often observed between C IV and He II $\lambda 1640$ (see Fine et al. 2010; Assef et al. 2011; Denney et al. 2013). We then fit the $\mathrm{C}$ IV emission line with sixth-order GaussHermite $(\mathrm{GH})$ polynomials that adopt the functional forms of, e.g., Cappellari et al. (2002) and the normalization of van der Marel \& Franx (1993). Iterative sigma clipping was employed in fitting both the continuum and the $\mathrm{C}$ IV emission line to exclude spurious flux contributions to the fit, typically from noise, poorly subtracted skyline residuals, or narrow, unmasked absorption features.

We prefer the $\mathrm{GH}$ polynomial fits for $\mathrm{C}$ IV because they tend to model the typically asymmetric and non-Gaussian profile of $\mathrm{C}$ IV better with fewer components than required when using (for example) only Gaussian functions. However, it is possible that in low-S/N data and in the presence of absorption, the freedom of the $\mathrm{GH}$ polynomials may lead to fit profiles with more structure than is likely real, since a lower $\chi^{2}$ can be achieved by matching the fit to what are ultimately spurious emission signatures ascribable to noise. Nonetheless, as high$\mathrm{S} / \mathrm{N}$ quasar spectra show that emission lines are not necessarily well described by any mathematically simple function, fitting the data with a more structured functional form may produce similar discrepancies owing to a lack of flexibility, i.e., underfitting the line.

As a test case to highlight the differences between our approach here and another typical approach of using multiple Gaussian functions, we have fit the four example spectra in Figure 3 with both GH polynomials (red curves) and multiple Gaussian functions (cyan curves). We have also done so to the coadded spectra of the 50 additional objects whose coadded spectra with GH fits are included as supplemental figures in the online journal. Each spectrum was fit with only two Gaussians (e.g., Shen et al. 2016b). While the multi-Gaussian fits to the SE spectra shown in Figure 3 are, indeed, more well behaved (have fewer "wiggles") than the GH fits, the reduced $\chi^{2}$ values, calculated excluding masked pixels, of the $\mathrm{GH}$ fits are marginally smaller than the multi-Gaussian fits to the SE spectra. Nonetheless, because $\chi_{\nu}^{2}<1.0$ in all cases, this is not terribly informative, but it is also clear from inspection of our SE spectra fits that bumps are often present coincident with the noise and not real features seen in the coadded spectra, so this overfitting is certainly present and a possible concern. On the other hand, for the two coadded spectra, the multi-Gaussian fits based on only two components appear to underfit the $\mathrm{C}_{\text {IV }}$ profile in the spectrum in the lower left panel, while arguably truncating the wings of the profile in the lower right panel, and the $\chi_{\nu}^{2}$ values of the $\mathrm{GH}$ fits are better than the multi-Gaussian fits-3.0 compared to 1.0 and 3.8 compared to 2.2 -for the Gaussian and GH fits in the left and right panels, respectively. This trend was also generally true for the 50 other sources that we fit with both functional forms; the two-component Gaussian fits produced lower $\chi_{\nu}^{2}$ values than the $\mathrm{GH}$ fits in only three cases. Visual inspection of the coadded spectra fits in the supplemental figure confirms that while sometimes the $\mathrm{GH}$ fits overfit the line, two-component Gaussians more often underfit 


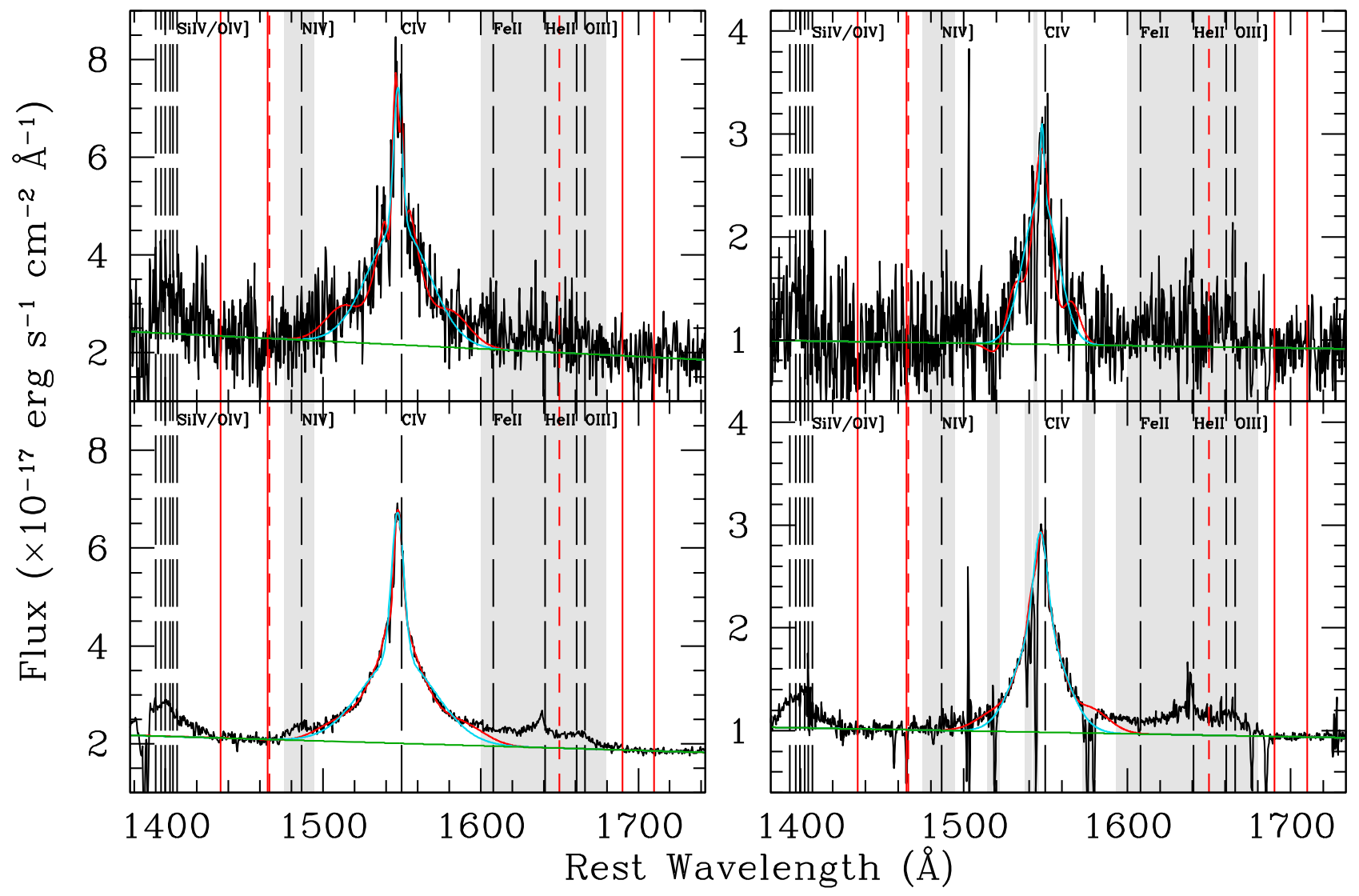

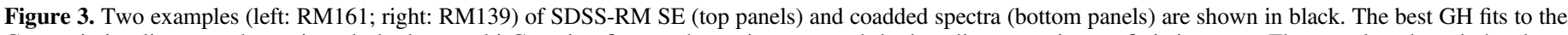

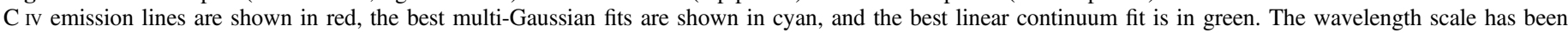

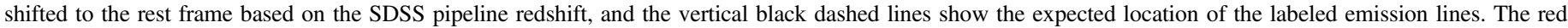

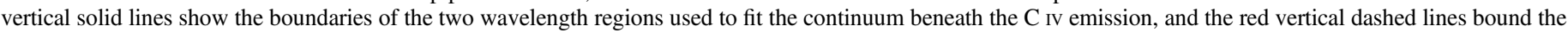

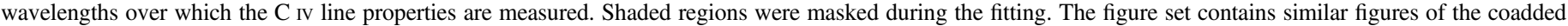
spectra and fits for 50 randomly selected objects from our sample.

(The complete figure set (50 images) is available.)

the line (RMID244 is a good example of this occurring simultaneously in the same object). These trends may have implications for comparisons of measured line properties between these two choices of fitting functions as a function of data quality or other line properties, and we further investigate this possibility below.

Spectra were fit interactively, but with an automated pipeline to better reproduce typical literature practices (e.g., Shen et al. 2008, 2011, 2016b). The automated pipeline set the red and blue continuum regions at the rest-frame wavelength ranges given above, although adjustments were made for the lowest-redshift objects where the blue region fell at the noisy edge of the spectrum, for the presence of significant absorption from inspection of the first-epoch SE spectrum or the coadded spectrum, or for chip defects in any spectrum. Next, the region over which $\mathrm{C}_{\text {IV }}$ was fit was set to rest-frame wavelengths $1466-1650 \AA$ A. This range was intentionally chosen to be broad enough to encompass all possible line widths. The $\mathrm{C}_{\text {IV }}$ line properties are measured directly from the best-fit profile, so the usual blended emission that may fall within these wavelengths, such as from $\mathrm{N}$ IV] or He II, is masked during fitting and does not contribute to the final fit. We added fit components iteratively, only adding additional components when residual emission line flux from any part of the line-usually the peak -remained above the average continuum noise level after subtracting each fit component. We do not ascribe physical meaning to any of the fit components. We simply aim to reconstruct the best overall model of the intrinsic, unblended, unabsorbed profile.

Manual intervention in the fitting procedure was necessary for several reasons. First, fitting was interrupted to change or identify additional masking needs-most often for absorption. The identification and details of contaminating features are more easily discerned in higher- $\mathrm{S} / \mathrm{N}$ spectra, so a more handson approach to the fitting was allowed when the additional signal provided this information. As such, modifications to the boundaries and masks were optimized on an object-to-object basis. This was often possible only for the coadded spectra, but when applied to the SE spectra, any masking or boundary changes were set by only the first-epoch spectrum and then kept the same for all additional epochs. Common modifications were to widen the red shelf mask further to the blue or the size and/or location of the $\mathrm{N}$ IV] and continuum masks to more optimal spectral regions. More detailed absorption-line masks were also often needed in higher-S/N spectra because weak absorption features were not always cleanly removed by the sigma-clipping procedure. Manual intervention was also used to confirm when multiple components were needed to fit the profile. This was done both for the coadded spectrum and for every SE spectrum. Finally, the interactive process also 
allowed us to flag and then remove previously unidentified "dropped" spectra from our analysis. These interactive steps likely increase the robustness of our fits compared to fully automated analyses of large survey samples, for which it is infeasible to visually inspect the fits to every spectrum.

To prevent any unconscious bias in the way the SE spectra were fit or masked for contaminating features, we fit the SE spectra before fitting the coadded spectra. To do otherwise might bias our analysis because using the information from the higher-S/N coadded spectra to inform treatment of the lower$\mathrm{S} / \mathrm{N}$ SE spectra is a luxury not afforded in typical analyses of survey-quality data.

\subsection{Measurements of CIV Emission Line Properties}

We focus our attention on only the two most commonly employed emission line width characterizations used to derive $\mathrm{BH}$ masses: the FWHM and the line dispersion $\left(\sigma_{l}\right)$, and the square root of the second moment of the line profile. However, we also consider the velocity width characterized by the mean absolute deviation $\left(\mathrm{MAD}^{17}\right)$ from the median velocity, defined following the notation of Peterson et al. (2004) as

$$
\mathrm{MAD}=\int\left|\lambda-\lambda_{\text {med }}\right| P(\lambda) d \lambda / \int P(\lambda) d \lambda,
$$

where $P(\lambda)$ is the continuum-subtracted emission-line profile and $\lambda_{\text {med }}$ is the flux-weighted median of the profile.

Additional properties of the $\mathrm{C}$ IV emission line we investigate include (i) its peak wavelength, defined from the centroid of the pixels $\geqslant 95 \%$ of the peak line flux; (ii) the EW, defined here with respect to the monochromatic continuum flux level measured in the continuum window covering rest frame $\sim 1450 \AA$; and the line "shape," characterized by (iii) the line kurtosis and (iv) the ratio FWHM $/ \sigma_{l}$. The amount by which the peak of $\mathrm{C}_{\mathrm{IV}}$ is blueshifted with respect to the systemic is often an additional line property of interest, but the determination of the blueshift depends on the reliability of the systemic redshift. Shen et al. (2016b) investigate the potential for biases in the redshifts determined for this sample, and we therefore defer discussions of the $\mathrm{C}$ IV blueshift to that work.

Line properties are measured from the continuum-subtracted best-fit GH polynomial fits. Any pixel with negative flux after the continuum subtraction is not included in the calculation of any line property. ${ }^{18}$ Individual spectra were not used when the measurement of any of these quantities was unreal, undefined, unphysical, etc. Examples of this include when an undefined EW measurement was returned because the mean continuum flux in the $1450 \AA$ region was found to be $\leqslant 0$, even if the fit found a weak line with a defined width, or when identification of the $\mathrm{C}$ IV emission line failed completely owing to low $\mathrm{S} / \mathrm{N}$, resulting in a null line width and other properties. After dropping all such cases, the subsequent analysis is based on the 482 coadded spectra and 15,275 SE spectra from all "good epochs."

\footnotetext{
17 We note that our definition of the MAD is not the same as the standard definition of the MAD as the median absolute deviation from the distribution median.

18 These are zero-noise profile fits. Negative pixels are therefore not due to noise characteristics, in which case they would be approximately balanced by positive pixels and their values should remain in the calculation. Instead, the fit sometimes produces negative pixels in masked regions (e.g., across absorption regions or in the CIV redshelf) where the profile is unconstrained Alternatively, it could occur in cases where absorption was not accurately masked owing to noise (see, e.g., the top right panel of Figure 3).
}

Similarly to the SE spectra S/N measurements, the SE line property "measurement" for each quasar is taken to be the median of the distribution of all the measurements from all good epochs of that source, and the measurement uncertainty is taken as the HIPR of the distribution. While all spectra nominally represent equivalent and independent observations of each source, we use the median and HIPR as opposed to the mean and standard deviation of these distributions to help account for possible outliers due to variable observing conditions between epochs. The emission-line property measurements for all objects based on the coadded spectra are made directly from the profile fit to the coadded spectrum for that source. The uncertainties in these measurements are estimated by performing Monte Carlo simulations using the fit to the coadded spectrum. We create 500 mock spectra by resampling the flux in the GH polynomial fit based on Gaussian deviates derived from the coadded error spectrum for each source. We assign the uncertainty in the measured line properties of each coadded spectrum to be the standard deviation about the mean of the distribution of 500 measurements of that property made from the mock spectra.

Figure 4 shows the relative line-width uncertainties, $\sigma_{V} / V$, for the FWHM and $\sigma_{l}$ of the SE and coadded spectra with respect to a line $\mathrm{S} / \mathrm{N}$ approximated as $C_{\text {win }} \mathrm{S} / \mathrm{N} \sqrt{\mathrm{EW} / W_{C}}$, given that the line photons dominate the continuum photons, where $W_{C}$ is the width of the continuum window over which the $\mathrm{S} / \mathrm{N}$ was measured, and $C_{\text {win }}$ is a normalization factor related to the sizes of the continuum and line integration windows and the EW of the line. We find that line-width uncertainties can be a significant fraction of the measured line width in the lowest-S/N spectra, and there is a factor of 2-3 increase in the magnitude of the statistical line-width measurement uncertainties between high-quality $(\mathrm{S} / \mathrm{N}>10)$ and lowquality $(\mathrm{S} / \mathrm{N}<5)$ data for all characterizations of the $\mathrm{C}$ IV velocity width. On the other hand, while the relative uncertainty drops to $\lesssim 5 \%$ for spectra with $\mathrm{S} / \mathrm{N} \gtrsim 10-20$, we find that the trend is shallower than the expected inverse linear relation slope of -1 . This is likely because of the details of the FWHM and $\sigma_{l}$ calculations. The FWHM does not depend on the whole line, but rather only on a fraction of the pixels (i.e., those near the peak and the $50 \%$ flux level). The shallower trend with $\sigma_{l}$ is likely because $\sigma_{l}$ is relatively more weighted by the flux in the wings of the line, where the line flux is lower. Table 1 lists the uncertainty distribution properties for the full sample and various subsamples divided by $\mathrm{S} / \mathrm{N}$ cuts.

\section{DISCUSSION}

We compare the line property measurements between the "highest-S/N" (coadded) spectra and the "lower-S/N" (SE) spectra by looking at the measurement differences, $\Delta(X)=X$ (coadded) - Median $[X(\mathrm{SE})]$, in the line property, $X$, as a function of data quality. Admittedly, the $\mathrm{S} / \mathrm{Ns}$ of a small percentage of the coadded spectra are also "low," compared to the median of the coadded spectrum $\mathrm{S} / \mathrm{N}$ distribution (see Figure 2), and fall within the lowest-quality subsample we consider in our analysis $(\mathrm{S} / \mathrm{N}<5)$. The coadded spectrum is, nonetheless, the highest-S/N observation of a given source and therefore always contains more information than an SE spectrum. We associate the SE S/N measurement, i.e., the median of the distribution of $\mathrm{S} / \mathrm{N}$ measurements from all $\mathrm{SE}$ spectra of a given object, with each $\Delta(X)$ value for that object to characterize the $\mathrm{S} / \mathrm{N}$ dependence of each property, $X$. We 


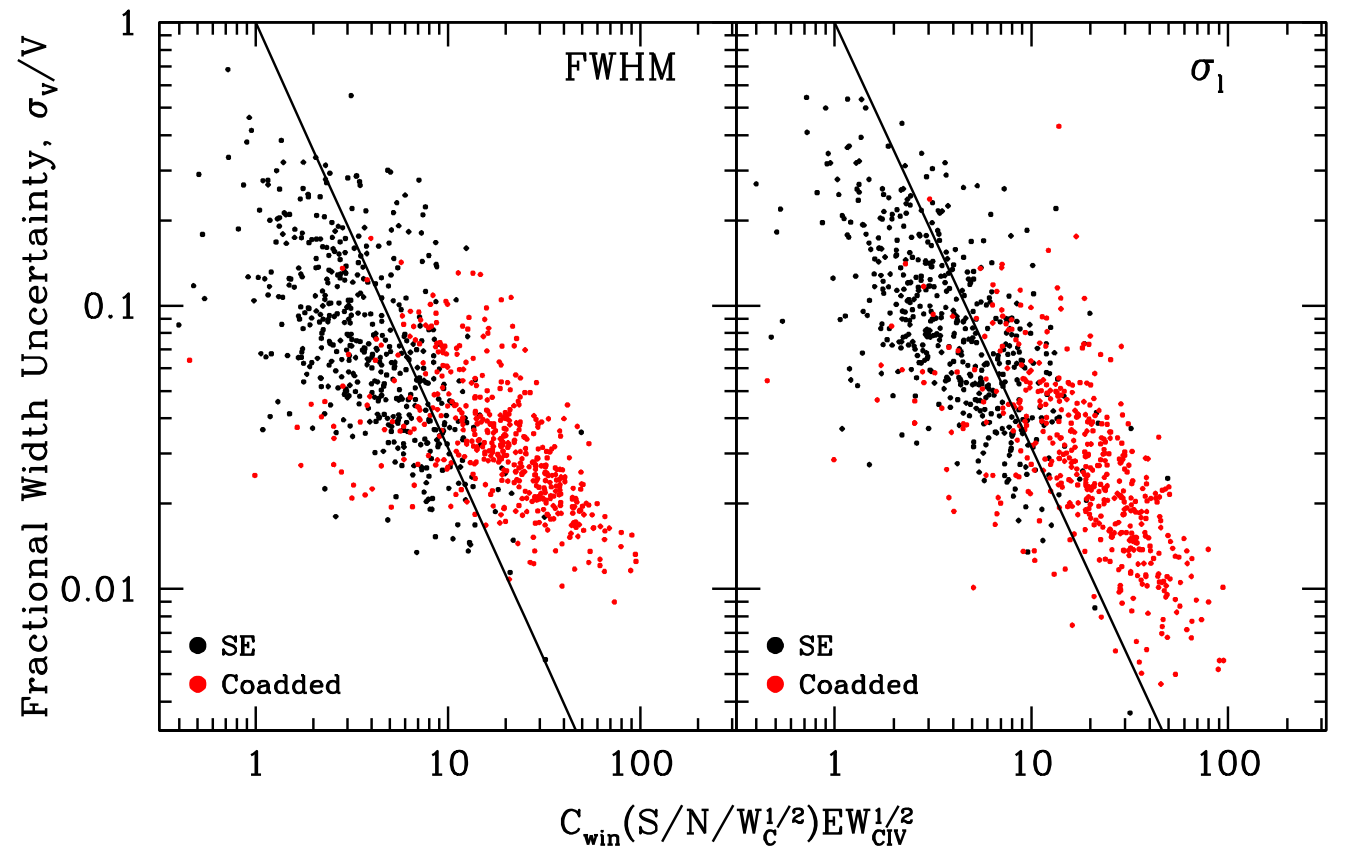

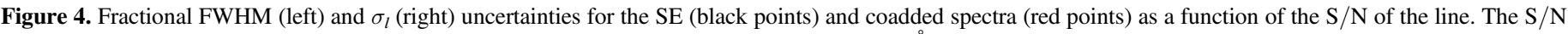

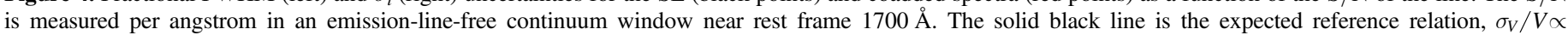
$\left(\mathrm{S} / \mathrm{N} \sqrt{\mathrm{EW} / W_{C}}\right)^{-1}$. See Section 3.2 for details.

further characterize this data-quality dependence on the line properties by looking at comparisons across subsamples with $\mathrm{S} / \mathrm{N}>10,<10$, and $<5$ based on the $\mathrm{SE} \mathrm{S} / \mathrm{N}$. Note that the objects in the $\mathrm{S} / \mathrm{N}<5$ subsample are contained in the $\mathrm{S} / \mathrm{N}<10$ subsample. The number of objects in the full sample and each subsample and the median $\mathrm{SE} S / \mathrm{N}$ within each sample are given in columns 3 and 4, respectively, of both Tables 1 and 2 .

\subsection{Data-quality Biases in Common Velocity Width Characterizations}

We first investigate how data quality affects the distributions of the fractional line-width differences, $\Delta(X) / X$ (coadded), for both the FWHM and line dispersion, $\sigma_{l}$. The left panel(s) in Figures 5 and 6 show the results for the fractional FWHM differences, $\Delta$ FWHM/FWHM. Table 2 gives the statistics of these distributions. We find a systematic bias such that the FWHM is overestimated in the SE spectra as compared to the coadded spectra. The magnitude of this bias, quantified by the median of the $\triangle \mathrm{FWHM} / \mathrm{FWHM}$ distributions, is within the line-width uncertainties only for the $\mathrm{SE} S / \mathrm{N}>10$ subsample, similar to other studies (Denney et al. 2009; Shen et al. 2011). While the bias is only marginally larger than the line-width uncertainties for the full sample and both subsamples with SE $\mathrm{S} / \mathrm{N}<10$, the distribution HIPR width, i.e., the scatter, increases by a factor of two from high to low $\mathrm{S} / \mathrm{N}$ and has a significant asymmetry that suggests that very large biases are possible, much larger than the formal measurement uncertainties, for some FWHM measurements (see the top left panel of Figure 5). Inspection of the left panel of Figure 6 demonstrates that this asymmetry and the magnitude of the systematic bias are anticorrelated with spectral $\mathrm{S} / \mathrm{N}$.

Interestingly, the results of Shen et al. (2011) for a different sample suggest that the FWHM in low-S/N data may be systematically underestimated when the profiles are described by multi-Gaussian fits, so details of the bias may depend on the specific functional form used to fit the data. Fortuitously, Shen et al. (2016b) completed a fully automated fitting pipeline analysis of this same data set that fits the C IV emission line with two Gaussian components and measured the FWHM. We therefore analyze the FWHM measurements from these multiGaussian fits to the SE and coadded spectra in the same way as we have for the $\mathrm{GH}$ fits in this work and make a direct comparison between these two independent methods, which is shown for the full sample and three $\mathrm{S} / \mathrm{N}$ subsamples in Figure 7 . We find that while the measurement uncertainties of the multi-Gaussian fits to the SE spectra (i.e., defined by the scatter in all SE measurements of each object) are 30\%-50\% larger than that of the $\mathrm{GH}$ polynomial fits at all $\mathrm{S} / \mathrm{N}$ levels, the distributions of $\triangle \mathrm{FWHM} / \mathrm{FWHM}$ appear more symmetric with smaller systematic differences between the $\mathrm{SE}$ and coadded measurements as a function of decreasing $\mathrm{S} / \mathrm{N}$.

As a means to understand these differences, we first directly compared the coadded FWHM measurements from both fitting methods. We find general agreement, but a linear regression analysis reveals a "twist" in the correlation compared to the unity relation-the LINMIX_ERR (Kelly 2007) best-fit slope is $0.898 \pm 0.019$. As a result, at small FWHM, the multiGaussian widths are larger than the $\mathrm{GH}$ fits and vice versa at large FWHM. Evidence from Section 3.2 (Figure 3) showing that the two-component Gaussian fits underfit the high-S/N profiles, while the $\mathrm{GH}$ fits more accurately reconstruct their intrinsic, non-Gaussianity, suggests to us that the bias in FWHM as a function of FWHM is in the Gaussian fits. It is likely attributable to the empirical observation that broad emission lines change "shape" as they broaden (see also discussion by Rafiee \& Hall 2011a, who find less biased Mg IIbased $\mathrm{BH}$ masses, overall, when using the line dispersion to characterize the broad-line velocity dispersion because of the change in line shape with FWHM). Regardless of the line profile characterization employed, this empirical change is 
Table 1

Data-quality Dependence of Distributions of SE C Iv Line Property Statistical Uncertainties

\begin{tabular}{|c|c|c|c|c|c|c|c|}
\hline $\begin{array}{l}\text { Distribution } \\
\text { Property } \\
\text { (1) }\end{array}$ & $\begin{array}{c}\text { Subsample } \\
\text { Description } \\
\text { (2) }\end{array}$ & $\begin{array}{c}\text { Number } \\
\text { of Obj. } \\
\text { (3) }\end{array}$ & $\begin{array}{c}\text { Median Sample } \\
\text { S/N } \\
(4)\end{array}$ & $\begin{array}{l}\text { Uncertainty } \\
\text { Median }^{\mathrm{b}} \\
\text { (5) }\end{array}$ & $\begin{array}{l}\text { Uncertainty } \\
\text { HIPR }^{\mathrm{b}} \\
\text { (6) }\end{array}$ & $\begin{array}{l}\text { Uncertainty } \\
\text { Mean }^{\mathrm{b}} \\
\text { (7) }\end{array}$ & $\begin{array}{l}\text { Uncertainty } \\
\text { Std. Dev. } \\
\text { (8) }\end{array}$ \\
\hline FWHM & $\mathrm{S} / \mathrm{N}>10$ & 122 & 13.9 & $186(4 \%)$ & $156(2 \%)$ & $276(5 \%)$ & $259(5 \%)$ \\
\hline FWHM & All & 482 & 5.5 & $340(7 \%)$ & $310(7 \%)$ & $454(10 \%)$ & $403(8 \%)$ \\
\hline FWHM & $\mathrm{S} / \mathrm{N}<10$ & 360 & 4.1 & $396(9 \%)$ & $328(7 \%)$ & $514(11 \%)$ & $425(8 \%)$ \\
\hline FWHM & $\mathrm{S} / \mathrm{N}<5$ & 217 & 2.9 & $498(11 \%)$ & $365(8 \%)$ & $602(13 \%)$ & $479(9 \%)$ \\
\hline Line dispersion, $\sigma_{l}$ & $\mathrm{~S} / \mathrm{N}>10$ & 122 & 13.9 & $185(5 \%)$ & $128(3 \%)$ & $247(6 \%)$ & $223(4 \%)$ \\
\hline Line dispersion, $\sigma_{l}$ & All & 482 & 5.5 & $285(8 \%)$ & $227(7 \%)$ & $379(11 \%)$ & $313(9 \%)$ \\
\hline Line dispersion, $\sigma_{l}$ & $\mathrm{~S} / \mathrm{N}<10$ & 360 & 4.1 & $321(9 \%)$ & $255(7 \%)$ & $424(12 \%)$ & $326(9 \%)$ \\
\hline Line dispersion, $\sigma_{l}$ & $\mathrm{~S} / \mathrm{N}<5$ & 217 & 2.9 & $368(11 \%)$ & $297(8 \%)$ & $481(15 \%)$ & $336(10 \%)$ \\
\hline MAD & $\mathrm{S} / \mathrm{N}>10$ & 122 & 13.9 & $133(5 \%)$ & $88(3 \%)$ & $176(6 \%)$ & $181(4 \%)$ \\
\hline MAD & All & 482 & 5.5 & $201(8 \%)$ & $175(6 \%)$ & $277(10 \%)$ & $251(9 \%)$ \\
\hline MAD & $\mathrm{S} / \mathrm{N}<10$ & 360 & 4.1 & $226(9 \%)$ & $187(7 \%)$ & $311(12 \%)$ & $262(9 \%)$ \\
\hline MAD & $\mathrm{S} / \mathrm{N}<5$ & 217 & 2.9 & $259(11 \%)$ & $212(8 \%)$ & $354(14 \%)$ & $267(10 \%)$ \\
\hline EW & $\mathrm{S} / \mathrm{N}>10$ & 122 & 13.9 & 7 & 3 & 9 & 7 \\
\hline EW & All & 482 & 5.5 & 17 & 11 & 27 & 39 \\
\hline EW & $\mathrm{S} / \mathrm{N}<10$ & 360 & 4.1 & 22 & 12 & 33 & 43 \\
\hline EW & $\mathrm{S} / \mathrm{N}<5$ & 217 & 2.9 & 30 & 14 & 43 & 53 \\
\hline Centroid & $\mathrm{S} / \mathrm{N}>10$ & 122 & 13.9 & 1.1 & 0.5 & 1.7 & 2.1 \\
\hline Centroid & All & 482 & 5.5 & 1.5 & 0.8 & 2.2 & 2.2 \\
\hline Centroid & $\mathrm{S} / \mathrm{N}<10$ & 360 & 4.1 & 1.8 & 0.9 & 2.4 & 2.3 \\
\hline Centroid & $\mathrm{S} / \mathrm{N}<5$ & 217 & 2.9 & 2.0 & 1.1 & 2.7 & 2.5 \\
\hline Kurtosis & $\mathrm{S} / \mathrm{N}>10$ & 122 & 13.9 & 0.12 & 0.05 & 0.14 & 0.09 \\
\hline Kurtosis & All & 482 & 5.5 & 0.17 & 0.08 & 0.20 & 0.14 \\
\hline Kurtosis & $\mathrm{S} / \mathrm{N}<10$ & 360 & 4.1 & 0.19 & 0.09 & 0.22 & 0.15 \\
\hline Kurtosis & $\mathrm{S} / \mathrm{N}<5$ & 217 & 2.9 & 0.22 & 0.10 & 0.25 & 0.16 \\
\hline Shape $\left(\mathrm{FWHM} / \sigma_{l}\right)$ & $\mathrm{S} / \mathrm{N}>10$ & 122 & 13.9 & 0.09 & 0.04 & 0.11 & 0.09 \\
\hline Shape $\left(\mathrm{FWHM} / \sigma_{l}\right)$ & All & 482 & 5.5 & 0.15 & 0.09 & 0.20 & 0.17 \\
\hline Shape $\left(\mathrm{FWHM} / \sigma_{l}\right)$ & $\mathrm{S} / \mathrm{N}<10$ & 360 & 4.1 & 0.18 & 0.09 & 0.24 & 0.18 \\
\hline Shape $\left(\mathrm{FWHM} / \sigma_{l}\right)$ & $\mathrm{S} / \mathrm{N}<5$ & 217 & 2.9 & 0.22 & 0.11 & 0.28 & 0.20 \\
\hline
\end{tabular}

Notes.

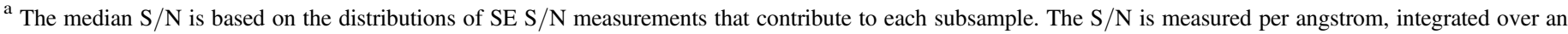
emission-line-free continuum window, $\Delta W$, covering many resolution elements near rest frame $1700 \AA$.

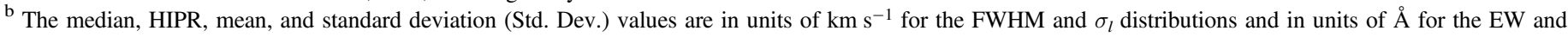

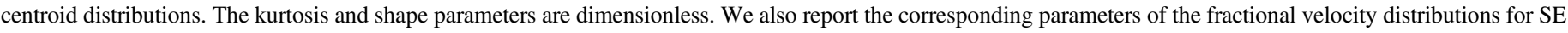
line-width uncertainties in parentheses to be consistent with what is shown in Figure 4.

more difficult to accurately characterize when limiting the profile fits to only two Gaussian components. The observed bias would presumably decrease by increasing the number of Gaussian components in order to more accurately fit the intrinsic non-Gaussianity of broad-line profiles.

The presence of this bias due to the two-component Gaussian fits underfitting the coadded spectrum profile prompts a final comparison, shown in Figure 8. Assuming again that the $\mathrm{GH}$ fits to the coadded spectrum provide a closer approximation to the intrinsic profile, we look at the FWHM difference between the SE two-component Gaussian fit and the coadded spectrum GH fit, i.e., (FWHM $\left.{ }_{\mathrm{GHcoadd}}-\mathrm{FWHM}_{\mathrm{GaussSE}}\right) /$ $\mathrm{FWHM}_{\mathrm{GHcoadd}}$ (black histograms), and the FWHM difference between the SE spectrum fit from both methods (red histograms). We find that (i) there is again an increasing systematic offset with decreasing $\mathrm{S} / \mathrm{N}$ between the $\mathrm{SE}$ and coadded spectra FWHM, and (ii) there is general consistency between the SE FWHM measurements made between the twocomponent Gaussian fits and the GH fits, albeit with a marginal bias in the sense that GH SE FWHM is slightly larger than twocomponent Gaussian FWHM.

We interpret the former observation (observation (i)) as a consequence of the two-component Gaussians underfitting the
high-S/N profile. As $\mathrm{S} / \mathrm{N}$ decreases, detailed information about the line profile is lost, which causes the increasing systematic bias in FWHM seen here and in Figure 5. However, two-component Gaussians that underfit the high-S/N profile never record this detailed information; thus, a signature of its loss in the form of a systematic offset with decreasing $\mathrm{S} / \mathrm{N}$ is not seen (Figure 7) because it was never there to begin with. However, the systematic offset seen with decreasing $\mathrm{S} / \mathrm{N}$ in the GH fits (Figure 5, left) is likely exacerbated by the flexibility of the $\mathrm{GH}$ profiles at low $\mathrm{S} / \mathrm{N}$, where they are more likely to overfit the line, leading to observation (ii). This suggests that two-component Gaussians are sufficient at low $\mathrm{S} / \mathrm{N}$, where the detailed profile information is already lost in the noise; thus, the flexibility of the GH polynomials is likely unwarranted. A more in-depth investigation into the differences between $\mathrm{GH}$ and multi-Gaussian fits on all line-width characterizations discussed here, though certainly of interest in this context, warrants its own thorough investigation and, as such, is outside the scope of the current work.

The right panel in Figures 5 and 6 and the statistics in Table 2 show the results for the line dispersion, $\Delta \sigma_{l} / \sigma_{l}$. A small systematic bias also exists in the line dispersion measurements, but in the opposite sense-the line dispersion 
Table 2

Data-quality Dependence of Distributions of $\mathrm{C}$ iv Line Property Biases, $\Delta X=X$ (coadded) $-X(\mathrm{SE})$

\begin{tabular}{|c|c|c|c|c|c|c|c|}
\hline $\begin{array}{l}\Delta X \text { Distribution } \\
\text { Property } \\
\text { (1) }\end{array}$ & $\begin{array}{c}\text { Subsample } \\
\text { Description } \\
\text { (2) }\end{array}$ & $\begin{array}{c}\text { Number } \\
\text { of Obj. } \\
\text { (3) }\end{array}$ & $\begin{array}{c}\text { Median Sample } \\
\text { S } / \mathrm{N} \\
(4)\end{array}$ & $\begin{array}{l}\text { Distribution } \\
\text { Median }^{\mathrm{b}} \\
\text { (5) }\end{array}$ & $\begin{array}{l}\text { Distribution } \\
\text { HIPR }^{\mathrm{b}} \\
\text { (6) }\end{array}$ & $\begin{array}{l}\text { Distribution } \\
\text { Mean }^{\mathrm{b}} \\
\text { (7) }\end{array}$ & $\begin{array}{c}\text { Distribution } \\
\text { Std. Dev. } \\
\text { (8) }\end{array}$ \\
\hline$\Delta \mathrm{FWHM}$ & $\mathrm{S} / \mathrm{N}>10$ & 122 & 13.9 & $-115(-0.028)$ & $438(0.079)$ & $-307(-0.085)$ & $725(0.227)$ \\
\hline$\Delta \mathrm{FWHM}$ & All & 482 & 5.5 & $-384(-0.091)$ & $628(0.169)$ & $-541(-0.165)$ & $995(0.396)$ \\
\hline$\Delta \mathrm{FWHM}$ & $\mathrm{S} / \mathrm{N}<10$ & 360 & 4.1 & $-471(-0.125)$ & $718(0.197)$ & $-629(-0.197)$ & $1075(0.442)$ \\
\hline$\Delta \mathrm{FWHM}$ & $\mathrm{S} / \mathrm{N}<5$ & 217 & 2.9 & $-513(-0.142)$ & $811(0.225)$ & $-718(-0.236)$ & $1242(0.534)$ \\
\hline$\Delta \sigma_{l}$ & $\mathrm{~S} / \mathrm{N}>10$ & 122 & 13.9 & $132(0.033)$ & $547(0.127)$ & $117(0.014)$ & $862(0.234)$ \\
\hline$\Delta \sigma_{l}$ & All & 482 & 5.5 & $257(0.073)$ & $607(0.150)$ & $289(0.059)$ & $768(0.210)$ \\
\hline$\Delta \sigma_{l}$ & $\mathrm{~S} / \mathrm{N}<10$ & 360 & 4.1 & $294(0.081)$ & $613(0.153)$ & $347(0.073)$ & 725 (0.199) \\
\hline$\Delta \sigma_{l}$ & $\mathrm{~S} / \mathrm{N}<5$ & 217 & 2.9 & $381(0.104)$ & $638(0.150)$ & $403(0.084)$ & $708(0.201)$ \\
\hline$\triangle \mathrm{MAD}$ & $\mathrm{S} / \mathrm{N}>10$ & 122 & 13.9 & $94(0.029)$ & $309(0.105)$ & $31(-0.001)$ & $623(0.228)$ \\
\hline$\Delta \mathrm{MAD}$ & All & 482 & 5.5 & $135(0.046)$ & $363(0.127)$ & $133(0.034)$ & $525(0.198)$ \\
\hline$\triangle \mathrm{MAD}$ & $\mathrm{S} / \mathrm{N}<10$ & 360 & 4.1 & $147(0.053)$ & $384(0.131)$ & $166(0.045)$ & $484(0.186)$ \\
\hline$\Delta \mathrm{MAD}$ & $\mathrm{S} / \mathrm{N}<5$ & 217 & 2.9 & $188(0.072)$ & $408(0.129)$ & $187(0.049)$ & $484(0.196)$ \\
\hline$\Delta \mathrm{EW}$ & $\mathrm{S} / \mathrm{N}>10$ & 122 & 13.9 & 3 & 5 & 2 & 16 \\
\hline$\Delta \mathrm{EW}$ & All & 482 & 5.5 & 9 & 10 & 13 & 28 \\
\hline$\Delta \mathrm{EW}$ & $\mathrm{S} / \mathrm{N}<10$ & 360 & 4.1 & 11 & 13 & 17 & 30 \\
\hline$\Delta \mathrm{EW}$ & $\mathrm{S} / \mathrm{N}<5$ & 217 & 2.9 & 17 & 14 & 24 & 34 \\
\hline$\Delta$ Centroid & $\mathrm{S} / \mathrm{N}>10$ & 122 & 13.9 & 0.19 & 1.92 & 6.8 & 61.4 \\
\hline$\Delta$ Centroid & All & 482 & 5.5 & -0.03 & 1.71 & 0.10 & 4.44 \\
\hline$\Delta$ Centroid & $\mathrm{S} / \mathrm{N}<10$ & 360 & 4.1 & -0.20 & 1.78 & -0.28 & 4.62 \\
\hline$\Delta$ Centroid & $\mathrm{S} / \mathrm{N}<5$ & 217 & 2.9 & -0.22 & 1.65 & -0.48 & 4.95 \\
\hline$\Delta$ Kurtosis & $\mathrm{S} / \mathrm{N}>10$ & 122 & 13.9 & 0.32 & 0.36 & 0.39 & 0.59 \\
\hline$\Delta$ Kurtosis & All & 482 & 5.5 & 0.52 & 0.39 & 0.65 & 0.73 \\
\hline$\Delta$ Kurtosis & $\mathrm{S} / \mathrm{N}<10$ & 360 & 4.1 & 0.63 & 0.39 & 0.75 & 0.75 \\
\hline$\Delta$ Kurtosis & $\mathrm{S} / \mathrm{N}<5$ & 217 & 2.9 & 0.77 & 0.42 & 0.89 & 0.79 \\
\hline$\Delta$ Shape $\left(\mathrm{FWHM} / \sigma_{l}\right)$ & $\mathrm{S} / \mathrm{N}>10$ & 122 & 13.9 & -0.07 & 0.19 & -0.12 & 0.31 \\
\hline$\Delta$ Shape $\left(\mathrm{FWHM} / \sigma_{l}\right)$ & All & 482 & 5.5 & -0.22 & 0.24 & -0.23 & 0.34 \\
\hline$\Delta$ Shape $\left(\mathrm{FWHM} / \sigma_{l}\right)$ & $\mathrm{S} / \mathrm{N}<10$ & 360 & 4.1 & -0.29 & 0.24 & -0.27 & 0.34 \\
\hline$\Delta$ Shape $\left(\mathrm{FWHM} / \sigma_{l}\right)$ & $\mathrm{S} / \mathrm{N}<5$ & 217 & 2.9 & -0.36 & 0.24 & -0.31 & 0.34 \\
\hline
\end{tabular}

Notes.

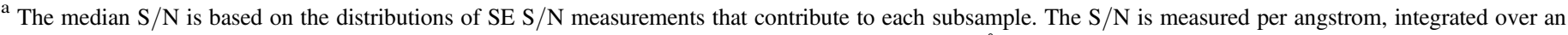
emission-line-free continuum window, $\Delta W$, covering many resolution elements near rest frame $1700 \AA$.

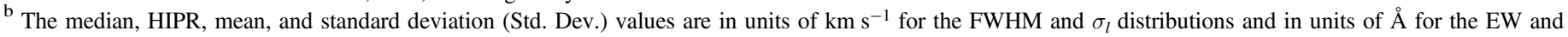

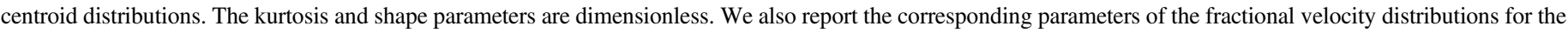
coadded-SE line widths, $\Delta \mathrm{FWHM} / \mathrm{FWHM}, \Delta \sigma_{l} / \sigma_{l}$, and $\Delta \mathrm{MAD} / \mathrm{MAD}$ in parentheses to be consistent with what is shown in Figures 5 and 9.

tends to be underestimated in lower-S/N spectra. The magnitude of this bias also increases with decreasing $\mathrm{S} / \mathrm{N}$. However, unlike the systematic bias in the FWHM measurements, the measurement uncertainties encompass the observed systematic shift in the SE line dispersion measurements at all spectral $\mathrm{S} / \mathrm{N}$ levels. The $\Delta \sigma_{l}$ distributions are also more symmetric than the $\triangle F W H M$ distributions, though the right panel of Figure 6 and statistics in Table 2 demonstrate that data quality still contributes to both the systematic bias and the broadening of the $\Delta \sigma_{l}$ distributions at lower $\mathrm{S} / \mathrm{N}$. We caution the reader, however, that these results hold only when line dispersion measurements are made selfconsistently across a sample. Larger DC offsets in line dispersion measurements, and thus $\mathrm{BH}$ mass estimates, can arise between samples that are analyzed with different spectral processing methods, i.e., continuum and line boundary placements and emission deblending assumptions (e.g., Denney et al. 2009; Fine et al. 2010; Assef et al. 2011). Such offsets can be surmounted by following the same spectral processing method used in the calibration of the desired mass scaling relationship (Vestergaard \& Peterson 2006; Park et al. 2013).

\subsection{Data-quality Biases in Other Line Properties}

We also investigate the data-quality dependence of the other line properties we measured above-the $\mathrm{C}$ IV line MAD, EW, shape (kurtosis and width ratio), and centroid (see Tables 1 and 2 ). The left panel of Figure 9 shows that the MAD behaves most similarly to $\sigma_{l}$ in that it increasingly underestimates the width with decreasing $\mathrm{S} / \mathrm{N}$, although the relative magnitude of the bias is smaller than that observed for both FWHM and $\sigma_{l}$, and the systematic offset stays well within the measurement uncertainties for all $\mathrm{S} / \mathrm{N}$ subsamples. The MAD number distributions as a function of $\triangle \mathrm{MAD}$ (right panel of Figure 9) are also relatively more symmetric with less extended wings in either direction compared to the FWHM and $\sigma_{l}$. This suggests that it may be a more robust characterization of the velocity width in low-quality data.

The trends with decreasing $\mathrm{S} / \mathrm{N}$ differ between the other three properties we investigated. The $\mathrm{C}$ IV line EW is systematically underestimated in the low-S/N spectra, but the degree of systematic bias is within the larger measurement uncertainties, which is consistent with the findings of Shen et al. (2011). For the $\mathrm{C}$ IV centroid, a factor of $\sim 2$ increase in statistical uncertainty is seen between the highest- and lowest-S/N 


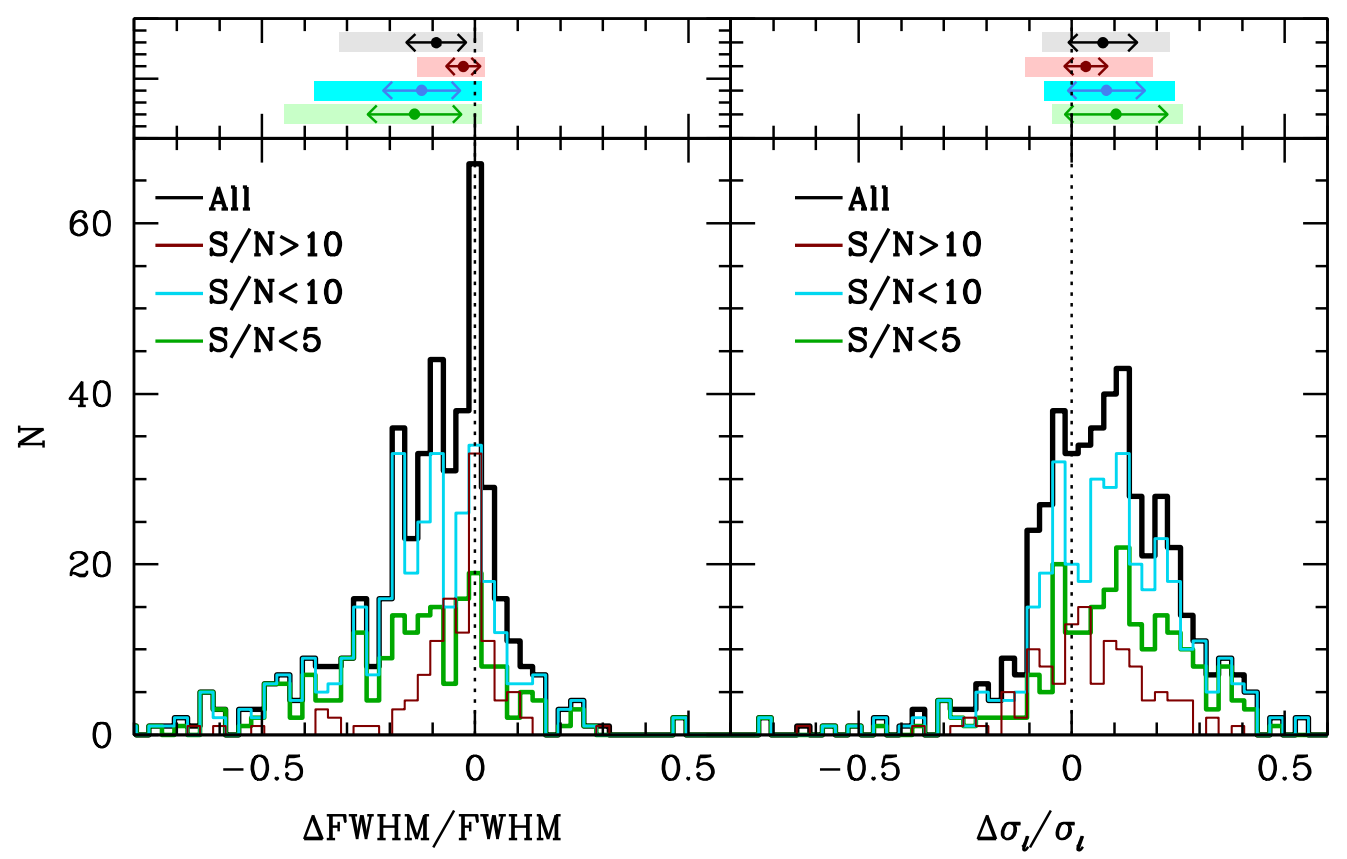

Figure 5. Histograms of the fractional differences between coadded and SE C IV emission line width measurements, FWHM (left) and $\sigma_{l}$ (right). The histograms are color-coded by data quality as shown in the legend (note: the $\mathrm{S} / \mathrm{N}<5$ subsample is contained within the $\mathrm{S} / \mathrm{N}<10$ subsample). The similarly color-coded shaded bars in the small top panels show the asymmetric scatter, given by the 16th-84th-percentile range, of each data-quality distribution, and the points and arrows correspond to the distribution median \pm the median fractional statistical uncertainty given in column 5 of Tables 1 and 2, respectively. An increasing bias is seen for lower-S/N subsamples that, while encompassed within the scatter of all distributions, is also encompassed within the statistical uncertainties at all $\mathrm{S} / \mathrm{N}$ levels for $\sigma_{l}$ but only for $\mathrm{S} / \mathrm{N}>10$ for the FWHM.

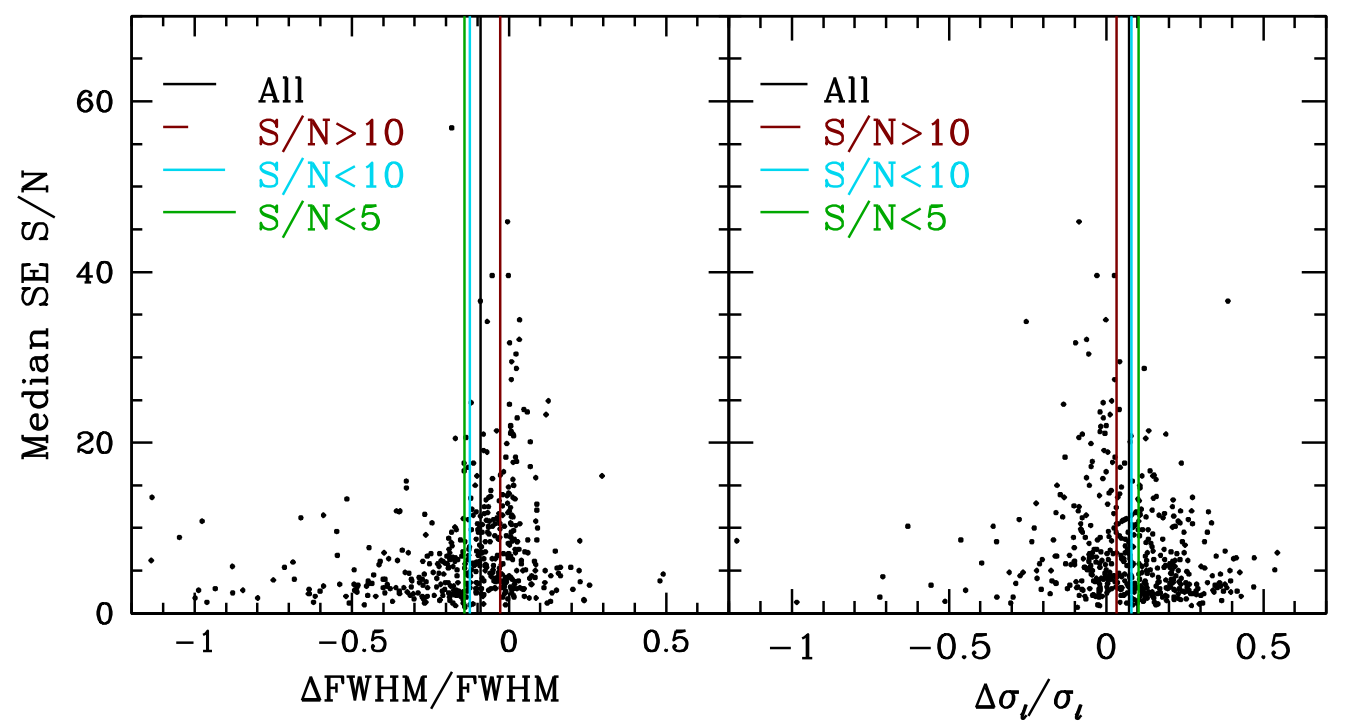

Figure 6. Distribution of data-quality-related biases in the line-width measurements. The left (right) panel shows the SE S/N as a function of the fractional difference between the coadded and SE FWHM (line dispersion, $\sigma_{l}$ ) of the $\mathrm{C}$ IV emission line. The vertical lines show the median velocity width difference of each histogram with the same color shown in Figure 5 and shown in the legend, and the length of the lines within the legend represents the HIPR range of each respective distribution.

subsamples, but a significant systematic bias is not detected for any $\mathrm{S} / \mathrm{N}$ subsample, demonstrating it to be the most robust of all properties we investigated in this respect (see Shen et al. $2016 \mathrm{~b}$ for similar results using this sample). The kurtosis of the $\mathrm{C}$ Iv line, however, is the least robust, which is not altogether surprising given its construction from relatively higher moments of the line profile. The formal measurement uncertainty only increases marginally with decreasing $\mathrm{S} / \mathrm{N}$, but there is a statistically significant $(>3 \sigma)$ systematic bias in $\Delta$ Kurtosis for all $\mathrm{S} / \mathrm{N}$ subsamples we investigate. This bias improves somewhat when we characterize the $\mathrm{C}$ IV profile by the FWHM $/ \sigma_{l}$ ratio. The measurement uncertainties increase by the same amount as the line-width uncertainties-a factor of $\sim 2-3$ between the $\mathrm{S} / \mathrm{N}>10$ and $\mathrm{S} / \mathrm{N}<5$ subsamples-as expected by construction, and the shape is systematically overestimated in lower- $\mathrm{S} / \mathrm{N}$ spectra. This bias is within the $1 \sigma$ measurement uncertainties of only the $\mathrm{S} / \mathrm{N}>10$ subsample but still within $\sim 1.5 \sigma$ for lower $\mathrm{S} / \mathrm{N}$, demonstrating it to be a more robust way to characterize the $\mathrm{C}$ IV profile than the kurtosis.

We also investigate the possible dependence of the linewidth differences on other line properties. Figure 10 shows the dependence of $\Delta$ FWHM (top panels) and $\Delta \sigma_{l}$ (bottom panels) 


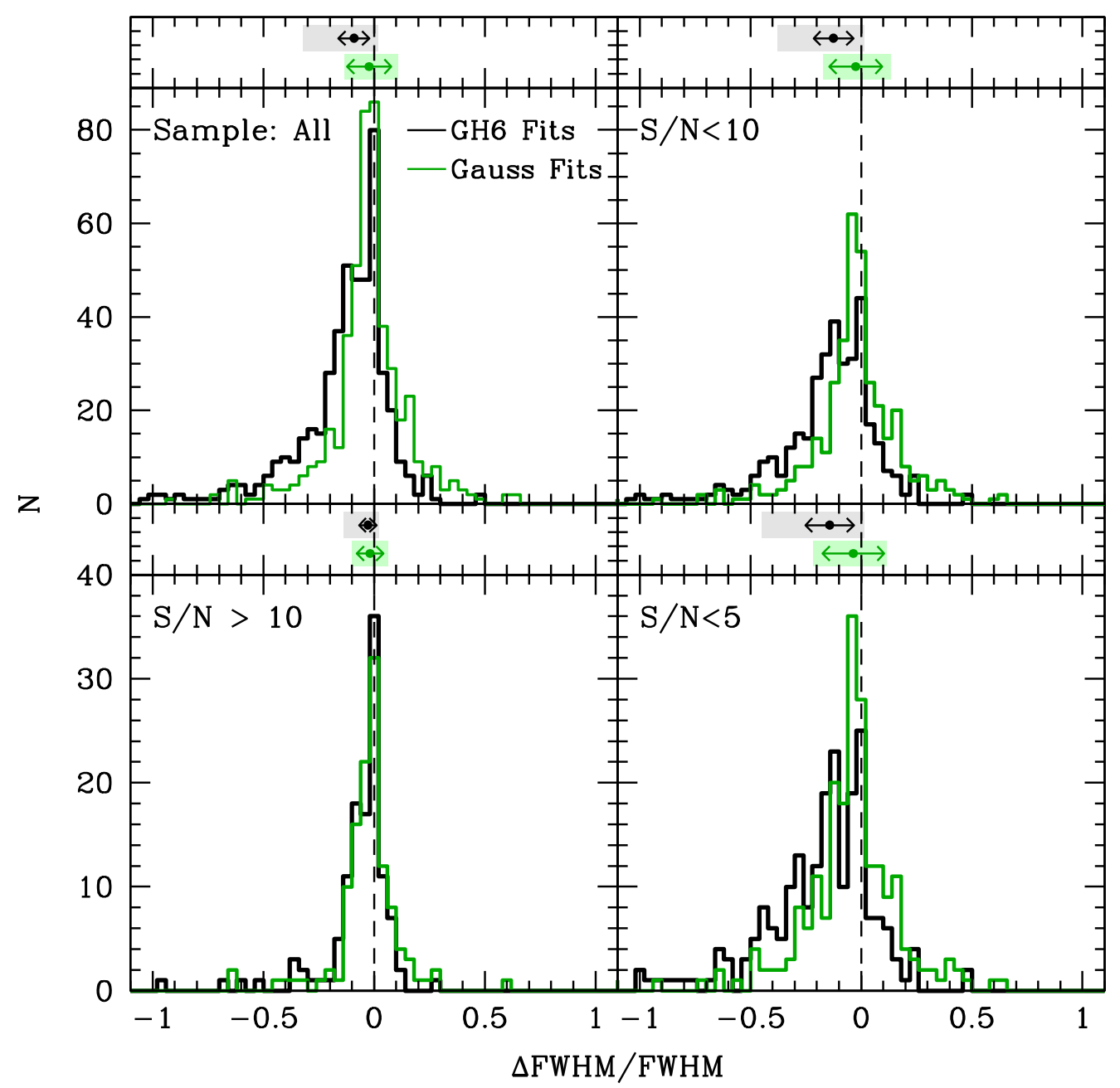

Figure 7. Left: same as the left panel of Figure 5, but comparing our GH polynomial fits to the two-component Gaussian fits of Shen et al. (2016b) made with a fully automated pipeline fitting procedure applied to the same data.

on the C Iv EW (left), kurtosis (middle), and the FWHM $/ \sigma_{l}$ ratio (right). We performed a formal linear regression analysis on the dependencies of the full sample using LINMIX_ERR (Kelly 2007) and quantify the results with a Spearman rankorder test. We evaluate the significance of any correlations using the Spearman rank correlation coefficient, $r_{s}$, the probability that a correlation is found by chance, $P_{\mathrm{ran}}$, and the formal uncertainties on the slope of the regression fit.

The only statistically significant trend with $\triangle$ FWHM is with the FWHM $/ \sigma_{l}$ ratio, but the correlation slope is consistent with a slope of zero within the $3 \sigma$ level. The correlation appears primarily driven by the large scatter seen only at small values of the FWHM $/ \sigma_{l}$ ratio. Direct inspection of the data (see, e.g., Figure 3) suggests that the main driver for the systematic overestimation of the FWHM in lower-S/N data is an underestimation of the emission line peak due to noise. This is also consistent with the findings of Denney et al. (2009) for $\mathrm{H} \beta$ FWHM measurements. With $\mathrm{C}$ iv, however, additional systematics contribute at low $\mathrm{S} / \mathrm{N}$ in cases where absorption cannot be accurately characterized. The peak and/or halfmaximum flux width can thus be under- or overestimated, on a case-dependent basis, leading to additional dispersion in the $\triangle$ FWHM distribution that may obscure other more subtle trends.
The trends in Figure 10 between $\Delta \sigma_{l}$ and the other $\mathrm{C}$ Iv line properties are all statistically significant and have regression fit slopes that deviate from zero by more than $3 \sigma$. There does not appear to be any strong dependence on data quality-from visual inspection of Figure 10, all data-quality subsamples generally follow the same trends. We focus additional attention on the correlation with the FWHM $/ \sigma_{l}$ ratio because it has the highest significance and the smallest scatter. We first investigate possible systematic differences between shape measured from the coadded versus SE spectrum. The top panel of Figure 11 suggests that objects can be roughly divided into (i) objects along the line of equal $\mathrm{SE}$ and coadded $\mathrm{C}$ IV shape, regardless of $\mathrm{S} / \mathrm{N}$, and (ii) a cloud of points with significantly different SE and coadded shape measurements, although there are points scattered between the two groups for low values of the coadded shape, and the two groups merge for large shape values. The latter population is dominated by the lowest-S/N subsample, but still includes some objects with reasonably high $\mathrm{S} / \mathrm{N}$.

We separate these two populations in the middle panel of Figure 11 by color-coding them by their deviation from having the same shape measurements. The bottom panel of Figure 11 shows this same population division in the $\Delta \sigma_{l}$-coadded $\mathrm{C}$ IV shape parameter space (same as the bottom right panel of Figure 10). This division demonstrates that the observed trend 


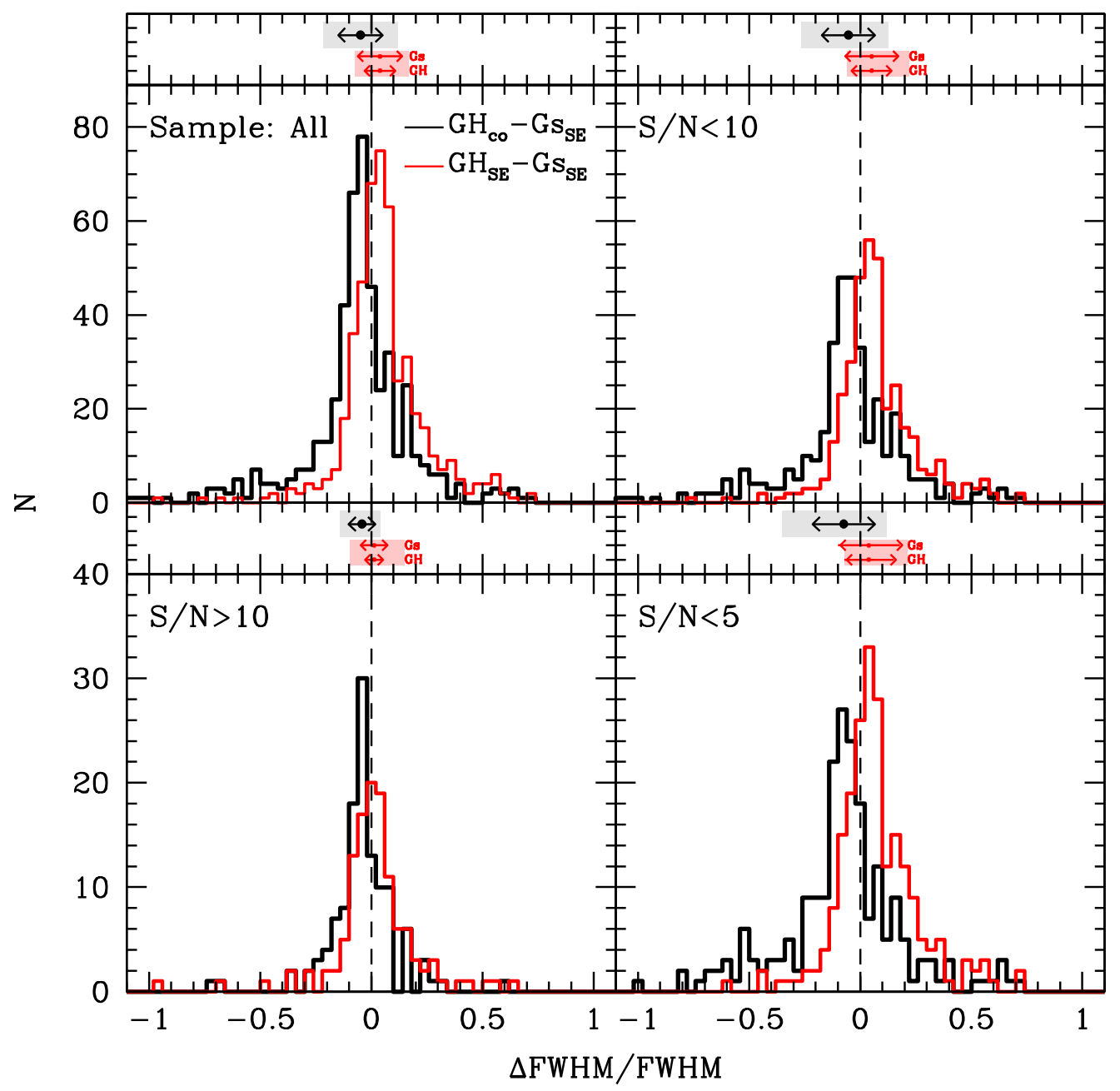

Figure 8. Left: same as Figure 7, but comparing the two-component Gaussian fits to the SE spectra of Shen et al. (2016b) to our GH polynomial fits to the coadded spectra (black histograms) and the SE spectra (red histograms). The length of the red arrows in the small top panels for each set of histograms represents the SE measurement uncertainties of the two-component Gaussian (Gs; top arrow) fits and the GH polynomial (GH; bottom arrow) fits, and the length of the black arrow also represents the measurement uncertainty of the SE measurements of the two-component Gaussian fits.

is due to objects for which the $\mathrm{C}$ IV profile is not accurately characterizable once the $\mathrm{S} / \mathrm{N}$ is degraded, even marginally, as we see deviations of objects from all $\mathrm{S} / \mathrm{N}$ subsamples, but this is a problem for only some quasars, which leads to the relatively larger scatter for smaller $\mathrm{FWHM} / \sigma_{l}$ ratios.

Inspection of individual cases suggests that this bias (and therefore the population separation with shape) occurs when the wings of the emission line become mischaracterized because of contamination from the noise of the continuum. The predominant effect is that the profile defined by the GH polynomial fits becomes truncated at artificially smaller zerointensity widths than inferred from the higher-S/N coadded spectrum. It is also possible that the continuum level is more accurately characterized in higher- $\mathrm{S} / \mathrm{N}$ data, leading to lower overall accuracy of the profile fit in the SE spectrum as compared to the coadded. This former effect can clearly be seen in the example spectra shown in the left panels of Figure 3. Another possible contributor to artificially truncated profile fits is blending of the $\mathrm{C}_{\text {IV }}$ wings with the red shelf of $\mathrm{C}_{\text {IV. }}$. The profiles most affected by premature truncation due to both noise and blending are those with small values of the shape parameters, which corresponds to the profiles that are very "peaky" with strong narrow velocity cores and very broad wings. This bias is likely the driver for the trends between $\Delta \sigma_{l}$ and the other line properties shown in Figure 10, as well.

\section{SUMMARY}

We have investigated data-quality-related statistical uncertainties and systematic biases in spectroscopic properties measured for high-redshift quasars. We have been particularly interested in properties of the $\mathrm{C}$ IV emission line, as this line is of interest for high-redshift $\mathrm{BH}$ mass estimates. This investigation was only possible because of the availability of spectroscopic monitoring data taken by the SDSS-RM project. Taken individually, these spectra are representative of typical surveyquality data; however, coadding produces a higher- $\mathrm{S} / \mathrm{N}$ spectrum, which can be used to make direct comparisons of the measurements of spectral properties between low- and high-S/N spectra.

Our analysis shows that the statistical measurement uncertainties of all the $\mathrm{C}_{\mathrm{IV}}$ velocity width characterizations we consider (FWHM, $\sigma_{l}$, and MAD) increase significantly, by a factor of 2-3, when going from high- to low-quality data (see Table 1, columns 4 and 5, and Figure 4). The statistical uncertainty in the $\mathrm{BH}$ mass estimates, which depend on the line width squared, are therefore a factor of two larger than this. We 

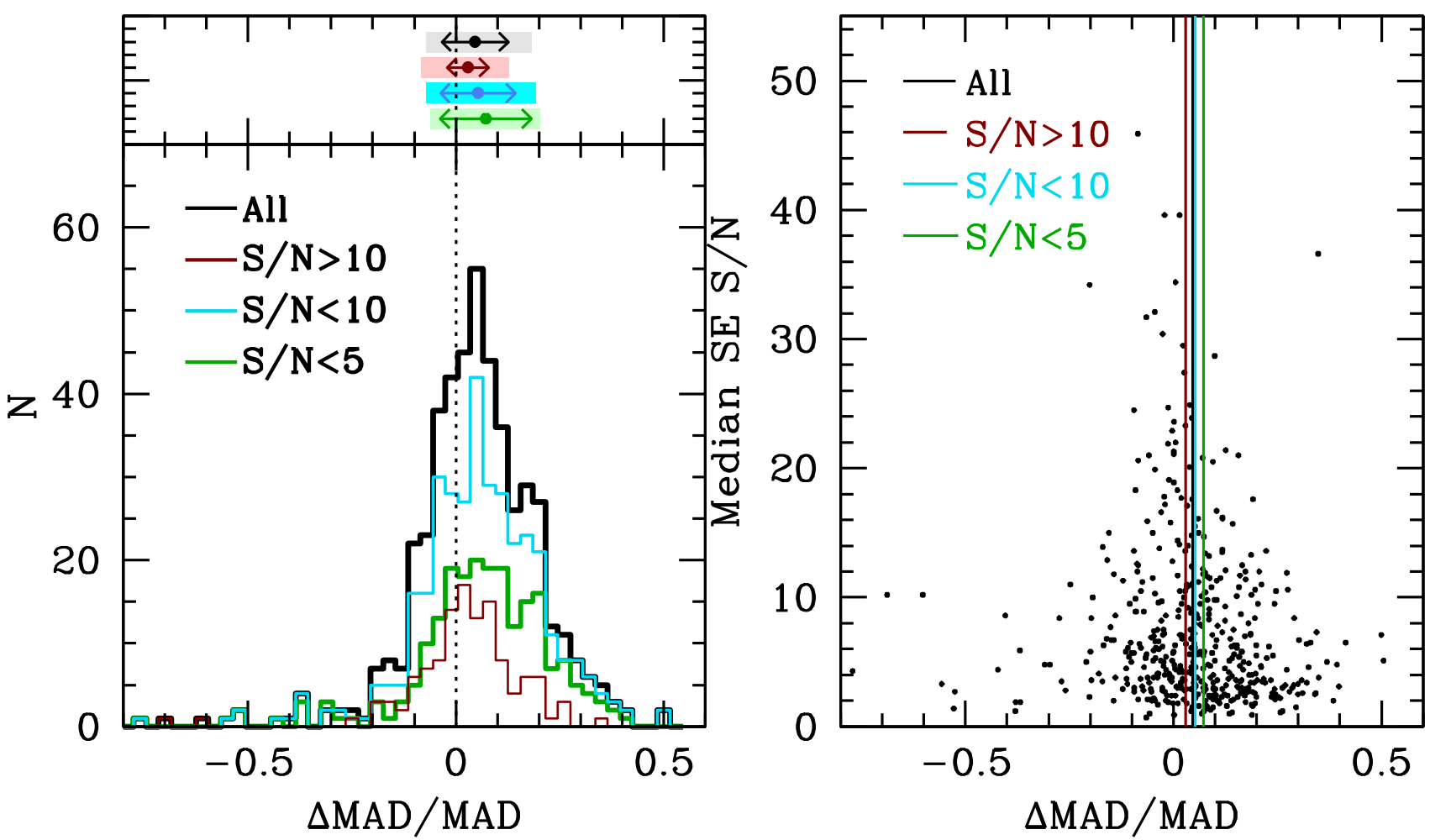

Figure 9. Left: same as Figure 5, but for the MAD. Right: same as Figure 6, but for the MAD.

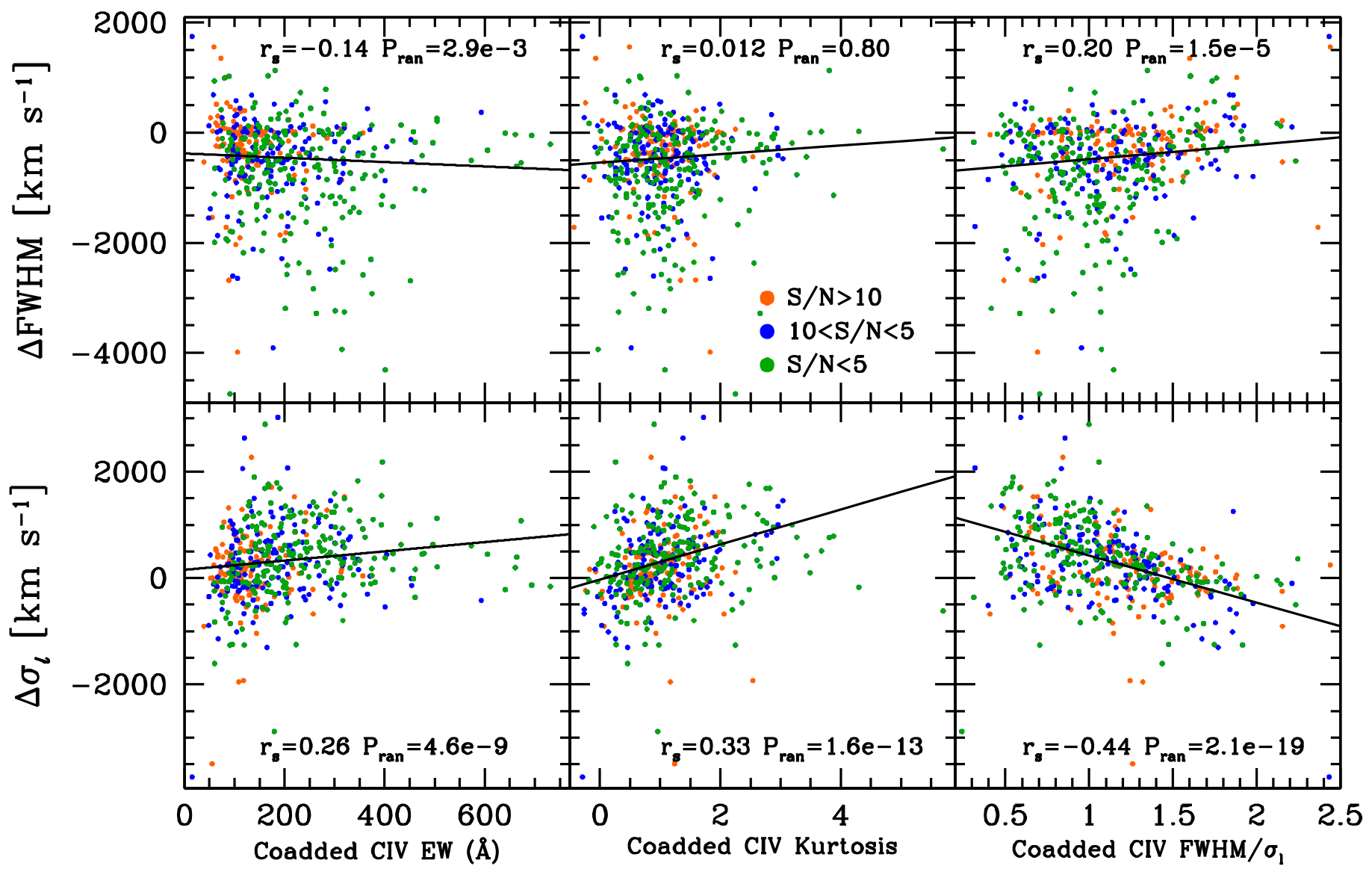

Figure 10. $\mathrm{C}$ iv line-width differences as a function of other $\mathrm{C}$ Iv emission line properties. The top (bottom) panels show results based on the FWHM (line dispersion, $\left.\sigma_{l}\right)$. The left, middle, and right panels show the dependence of the difference on the $\mathrm{C}$ Iv EW, kurtosis, and shape (FWHM/ $\left.\sigma_{l}\right)$, respectively. The points are colorcoded by the varying data quality of each subsample: $\mathrm{S} / \mathrm{N}>10$ (orange), $10<\mathrm{S} / \mathrm{N}<5$ (blue), and $\mathrm{S} / \mathrm{N}<5$ (green). The solid black lines show the best-fit linear regression fit. The Spearman rank correlation coefficient, $r_{s}$, and the probability that a correlation is found by chance, $P_{\text {ran }}$, are given in each panel. 


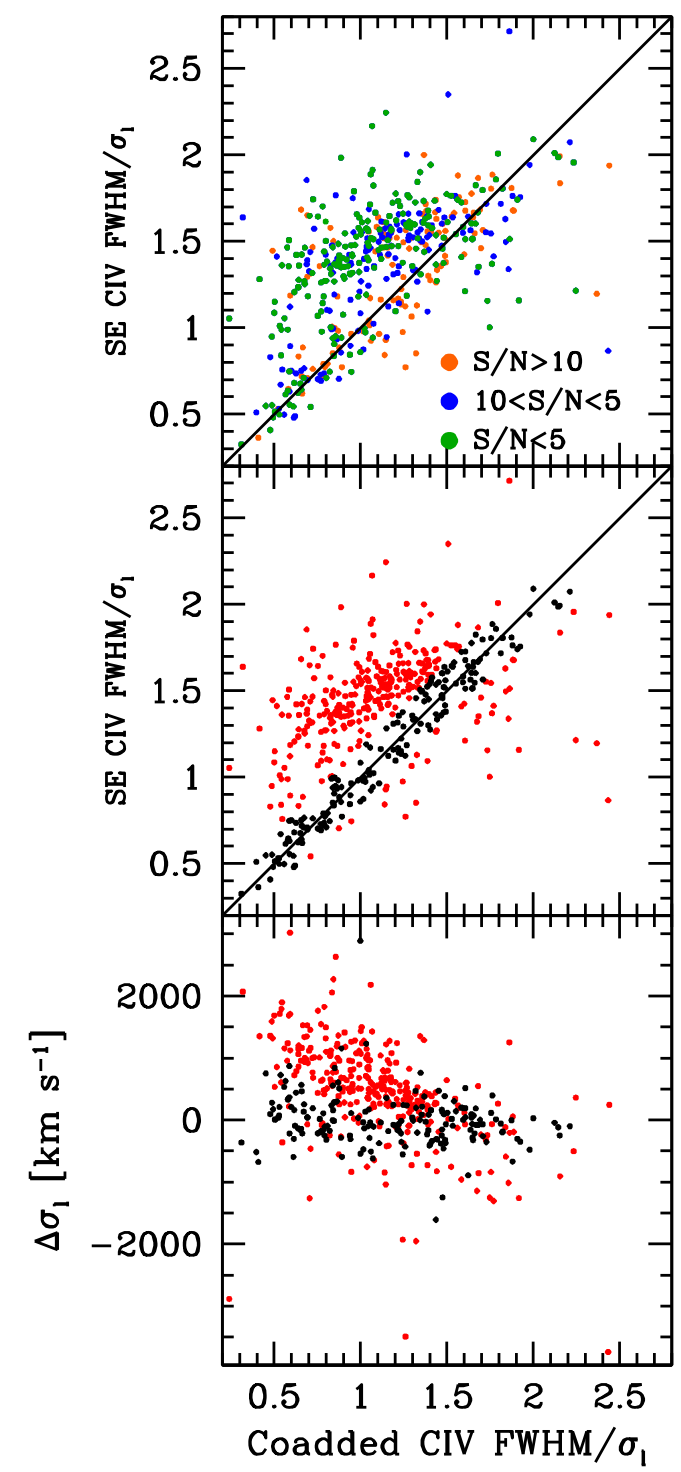

Figure 11. Trends between the $\mathrm{C}_{\mathrm{IV}}$ shape parameter $\left(\mathrm{FWHM} / \sigma_{l}\right)$ and the coadded-SE line dispersion differences. The top panel compares the SE C IV line shape measurements to those measured from the coadded spectra; colors represent different $\mathrm{S} / \mathrm{N}$ subsamples and are the same as in Figure 10. The middle panel is the same as the top panel, except the color coding now reflects the division of objects into two populations: the black (red) points represent objects for which the SE shape is (is not) roughly consistent with the coadded shape. The black points are defined by objects within the $1 \sigma$ scatter of having the same shape (black solid line), where $1 \sigma$ is defined from only points below this relation. All other objects are shown in red. The bottom panel is the same as the bottom right panel of Figure 10 but uses the same color coding as the middle panel.

also find the following systematic trends for the line-width characterizations we consider:

FWHM. At low $\mathrm{S} / \mathrm{N}$, a systematic overestimation of the $\mathrm{C}$ IV FWHM measurements is introduced, although it is not significantly larger than the measurement uncertainties, on average. The observed bias appears to be attributed to inaccuracies in characterizing the intrinsically non-Gaussian, complex line profile in noisy data (see Figure 6 and Table 2). Uncharacterized and/or peak absorption and the possibility that the "flexibility" of the GH polynomials is overfitting the profiles at low S/N likely exacerbate the FWHM biases for this fitting method (e.g., RMID 030, 602, and 740; see also Assef et al. 2011; Denney et al. 2013), and the systematic bias can be severe in some cases. This systematic bias is lessened when using multi-Gaussian fits to the low-S/N profiles instead of $\mathrm{GH}$ fits, at the expense of an $\sim 30 \%-50 \%$ increase in measurement uncertainties, because a two-component Gaussian fit is sufficient to fit the decreased amount of profile information available in the spectrum at these noise levels and the additional structure is less likely to overfit what is ultimately noise. Nonetheless, care should be taken in using only twocomponent Gaussians to fit relatively higher $\mathrm{S} / \mathrm{N}$ profiles because the differences between $\mathrm{GH}$ and multi-Gaussian widths measured from the high-S/N coadded spectra found as a function of FWHM suggest that a two-component Gaussian underfits the profile, which consequently overestimates (underestimates) the FWHM of relatively narrower (broader) lines, in addition to obscuring the presence of the systematic overestimation of the FWHM with decreasing $\mathrm{S} / \mathrm{N}$.

Line Dispersion. Biases in $\sigma_{l}$ at low $\mathrm{S} / \mathrm{N}$ are less than those in the FWHM and scale with the statistical uncertainty. When present, underestimation of $\sigma_{l}$ is caused mainly by the inability to accurately fit the emission-line wings in the presence of a noisy continuum. As a result, C IV profiles with "peaky" cores and extended wings are likely to be biased more significantly than "stumpy" or "boxy" profiles without extended wings (Figures 10 and 11).

$M A D$. The systematic bias in the MAD measurements was relatively smaller than for the FWHM and $\sigma_{l}$. The bias was also always well within the statistical uncertainties, and the distribution of $\triangle \mathrm{MAD}$ measurements remains relatively symmetric and centrally peaked with decreasing $\mathrm{S} / \mathrm{N}$ (see Figure 9).

These trends of FWHM and line dispersion measurements with $\mathrm{S} / \mathrm{N}$ when using $\mathrm{GH}$ functional fits are consistent with the results for $\mathrm{H} \beta$ from similar analyses presented by Denney et al. (2009), but that study did not investigate the MAD. We conclude here that the MAD is the most reliable measure of the velocity width for low-quality data. Nonetheless, further analysis is needed to investigate how good of a proxy this characterization is for the virial BLR velocity (see Peterson et al. 2004), and SE BH mass scaling relationships have not yet been developed and calibrated for this characterization.

We also stress to the reader that the present study has only been focused on biases due to data-quality considerations. We make no preference for which line-width characterization is a better proxy for the reverberating BLR velocity dispersion that traces the gravitational potential of the BH. The FWHM is often preferred because it is simpler to measure and less susceptible to the subjectiveness of deblending procedures. On the other hand, recent studies (Assef et al. 2011; Denney 2012; Denney et al. 2013) have demonstrated that $\sigma_{l}$ is less biased than FWHM for estimating $\mathrm{C}$ IV $\mathrm{BH}$ masses, which is least partially attributable to the presence of nonvariable flux contributions to the $\mathrm{C}$ IV emission line and/or a continuum color term that are yet unaccounted for in $\mathrm{C}$ IV-based $\mathrm{BH}$ mass scaling relation calibrations (see also Rafiee \& Hall 2011a, for similar Mg II trends, and Peterson 2014, for additional discussions).

We are grateful for the editorial contributions to this work from C. S. Kochanek. K.D.D. is supported by an NSF AAPF fellowship awarded under NSF grant AST-1302093. K.H. acknowledges support from STFC grant ST/M001296/1. W. 
N.B. acknowledges support from NSF grant AST-1516784. L. C.H. thanks Carnegie Observatories for providing telescope access and acknowledges financial support from Peking University, the Kavli Foundation, the Chinese Academy of Science through grant No. XDB09030102 (Emergence of Cosmological Structures) from the Strategic Priority Research Program, and the National Natural Science Foundation of China through grant No. 11473002. B.P.M. is grateful for support from NSF grant AST-10008882. Funding for SDSS-III has been provided by the Alfred P. Sloan Foundation, the Participating Institutions, the National Science Foundation, and the U.S. Department of Energy Office of Science. The SDSSIII Web site is http://www.sdss3.org/. SDSS-III is managed by the Astrophysical Research Consortium for the Participating Institutions of the SDSS-III Collaboration, including the University of Arizona, the Brazilian Participation Group, Brookhaven National Laboratory, University of Cambridge, Carnegie Mellon University, University of Florida, the French Participation Group, the German Participation Group, Harvard University, the Instituto de Astrofisica de Canarias, the Michigan State/Notre Dame/JINA Participation Group, Johns Hopkins University, Lawrence Berkeley National Laboratory, Max Planck Institute for Astrophysics, Max Planck Institute for Extraterrestrial Physics, New Mexico State University, New York University, Ohio State University, Pennsylvania State University, University of Portsmouth, Princeton University, the Spanish Participation Group, University of Tokyo, University of Utah, Vanderbilt University, University of Virginia, University of Washington, and Yale University.

\section{REFERENCES}

Assef, R. J., Denney, K. D., Kochanek, C. S., et al. 2011, ApJ, 742, 93 Baskin, A., \& Laor, A. 2005, MNRAS, 356, 1029

Bentz, M. C., Denney, K. D., Grier, C. J., et al. 2013, ApJ, 767, 149

Bentz, M. C., Peterson, B. M., Netzer, H., Pogge, R. W., \& Vestergaard, M. 2009a, ApJ, 697, 160

Bentz, M. C., Peterson, B. M., Pogge, R. W., \& Vestergaard, M. 2009b, ApJL, 694, L166

Blandford, R. D., \& McKee, C. F. 1982, ApJ, 255, 419

Cappellari, M., Verolme, E. K., van der Marel, R. P., et al. 2002, ApJ, 578, 787

Collin, S., Kawaguchi, T., Peterson, B. M., \& Vestergaard, M. 2006, A\&A, 456, 75

Dawson, K. S., Schlegel, D. J., Ahn, C. P., et al. 2013, AJ, 145, 10

Debuhr, J., Quataert, E., \& Ma, C.-P. 2011, MNRAS, 412, 1341

Denney, K. D. 2012, ApJ, 759, 44

Denney, K. D., Peterson, B. M., Dietrich, M., Vestergaard, M., \& Bentz, M. C. 2009, ApJ, 692, 246

Denney, K. D., Pogge, R. W., Assef, R. J., et al. 2013, ApJ, 775, 60

Eisenstein, D. J., Weinberg, D. H., Agol, E., et al. 2011, AJ, 142, 72

Fabian, A. C. 2012, ARA\&A, 50, 455

Ferrarese, L., \& Merritt, D. 2000, ApJL, 539, L9

Ferrarese, L., Pogge, R. W., Peterson, B. M., et al. 2001, ApJL, 555, L79

Fine, S., Croom, S. M., Bland-Hawthorn, J., et al. 2010, MNRAS, 409, 591

Gebhardt, K., Bender, R., Bower, G., et al. 2000a, ApJL, 539, L13

Gebhardt, K., Kormendy, J., Ho, L. C., et al. 2000b, ApJL, 543, L5
Graham, A. W. 2007, MNRAS, 379, 711

Graham, A. W., Onken, C. A., Athanassoula, E., \& Combes, F. 2011, MNRAS, 412, 2211

Grier, C. J., Peterson, B. M., Horne, K., et al. 2013, ApJ, 764, 47

Gunn, J. E., Siegmund, W. A., Mannery, E. J., et al. 2006, AJ, 131, 2332

Hopkins, P. F., \& Elvis, M. 2010, MNRAS, 401, 7

Jahnke, K., \& Macciò, A. V. 2011, ApJ, 734, 92

Kaspi, S., Smith, P. S., Netzer, H., et al. 2000, ApJ, 533, 631

Kelly, B. C. 2007, ApJ, 665, 1489

Kilerci Eser, E., Vestergaard, M., Peterson, B. M., Denney, K. D., \& Bentz, M. C. 2015, ApJ, 801, 8

Kormendy, J., \& Ho, L. C. 2013, ARA\&A, 51, 511

Kormendy, J., \& Richstone, D. 1995, ARA\&A, 33, 581

Laor, A. 1998, ApJL, 505, L83

Magorrian, J., Tremaine, S., Richstone, D., et al. 1998, AJ, 115, 2285

McConnell, N. J., \& Ma, C.-P. 2013, ApJ, 764, 184

McLure, R. J., \& Jarvis, M. J. 2002, MNRAS, 337, 109

Nelson, C. H., Green, R. F., Bower, G., Gebhardt, K., \& Weistrop, D. 2004, ApJ, 615, 652

Netzer, H., Lira, P., Trakhtenbrot, B., Shemmer, O., \& Cury, I. 2007, ApJ, 671,1256

Onken, C. A., Ferrarese, L., Merritt, D., et al. 2004, ApJ, 615, 645

Pâris, I., Petitjean, P., Aubourg, É., et al. 2014, A\&A, 563, A54

Park, D., Kelly, B. C., Woo, J.-H., \& Treu, T. 2012, ApJS, 203, 6

Park, D., Woo, J.-H., Denney, K. D., \& Shin, J. 2013, ApJ, 770, 87

Peterson, B. M. 1993, PASP, 105, 247

Peterson, B. M. 2014, SSRv, 183, 253

Peterson, B. M., Ferrarese, L., Gilbert, K. M., et al. 2004, ApJ, 613, 682

Rafiee, A., \& Hall, P. B. 2011a, MNRAS, 415, 2932

Rafiee, A., \& Hall, P. B. 2011b, ApJS, 194, 42

Runnoe, J. C., Brotherton, M. S., DiPompeo, M. A., \& Shang, Z. 2014, MNRAS, 438, 3263

Runnoe, J. C., Brotherton, M. S., Shang, Z., \& DiPompeo, M. A. 2013, MNRAS, 434, 848

Schmidt, M. 1963, Natur, 197, 1040

Schneider, D. P., Richards, G. T., Hall, P. B., et al. 2010, AJ, 139, 2360

Sergeev, S. G., Doroshenko, V. T., Dzyuba, S. A., et al. 2007, ApJ, 668, 708

Shen, Y., Greene, J. E., Strauss, M. A., Richards, G. T., \& Schneider, D. P. 2008, ApJ, 680, 169

Shen, Y., \& Liu, X. 2012, ApJ, 753, 125

Shen, Y., Brandt, W. N., Dawson, K. S., et al. 2015, ApJS, 216, 4

Shen, Y., Brandt, W. N., Denney, K. D., et al. 2016b, arXiv:1602.03894

Shen, Y., Horne, K., Grier, C. J., et al. 2016a, ApJ, 818, 30

Shen, Y., Richards, G. T., Strauss, M. A., et al. 2011, ApJS, 194, 45

Smee, S. A., Gunn, J. E., Uomoto, A., et al. 2013, AJ, 146, 32

Steinhardt, C. L., \& Elvis, M. 2010, MNRAS, 402, 2637

Sulentic, J. W., Bachev, R., Marziani, P., Negrete, C. A., \& Dultzin, D. 2007, ApJ, 666, 757

Sun, M., Trump, J. R., Brandt, W. N., et al. 2015a, ApJ, 802, 14

Sun, M., Trump, J. R., Shen, Y., et al. 2015b, ApJ, 811, 42

Tremaine, S., Gebhardt, K., Bender, R., et al. 2002, ApJ, 574, 740

Vanden Berk, D. E., Wilhite, B. C., Kron, R. G., et al. 2004, ApJ, 601, 692

van der Marel, R. P., \& Franx, M. 1993, ApJ, 407, 525

Vestergaard, M. 2002, ApJ, 571, 733

Vestergaard, M., \& Peterson, B. M. 2006, ApJ, 641, 689

Wandel, A. 2002, ApJ, 565, 762

Wandel, A., Peterson, B. M., \& Malkan, M. A. 1999, ApJ, 526, 579

Wang, J., Dong, X., Wang, T., et al. 2009, ApJ, 707, 1334

Woo, J., Treu, T., Barth, A. J., et al. 2010, ApJ, 716, 269

Woo, J.-H., Schulze, A., Park, D., et al. 2013, ApJ, 772, 49

York, D. G., Adelman, J., Anderson, J. E., Jr., et al. 2000, AJ, 120, 1579

Zu, Y., Kochanek, C. S., \& Peterson, B. M. 2011, ApJ, 735, 80 